\title{
The Price of a Smile: \\ Hedging and Spanning in Option Markets
}

\author{
Andrea Buraschi and Jens Jackwerth*
}

London Business School and University of Wisconsin at Madison

First Draft: November 12, 1997

This Draft: May 7, 2000

*Andrea Buraschi (corresponding author) is from London Business School, Sussex Place, Regent's Park, London NW1 4SA, United Kingdom. Tel: +44-20-7262-5050, fax: +44-20-7724-3317, aburaschi@lbs.ac.uk. Jens Jackwerth is from the University of Wisconsin at Madison, Finance Department, School of Business, 975 University Avenue, Madison, WI 53706, Tel: 608-262-6032, Fax: 608-265-4195, jjackwerth@bus.wisc.edu. For helpful comments on this paper we wish to thank Sudipto Bhattacharya, Mark Britten-Jones, Ian Cooper, the editor Bernard Dumas, Julian Franks, Erzo Luttmer, Davide Menini, Anthony Neuberger, Stephen Schaefer, an anonymous referee, and seminar participants at EFMA Lisbon, WFA Conference, 1998 Computational Finance Conference New York, The University of Chicago, HEC Business School, London Business School, London School of Economics, City University of London, Oxford Business School, Konstanz University, University of Wisconsin at Madison, Georgetown University. 
Abstract: The volatility smile changed drastically around the crash of 1987 and new option pricing models have been proposed in order to accommodate that change. Deterministic volatility models allow for more flexible volatility surfaces but refrain from introducing additional risk-factors. Thus, options are still redundant securities. Alternatively, stochastic models introduce additional risk-factors and options are then needed for spanning of the pricing kernel. We develop a statistical test based on this difference in spanning. Using daily S\&P500 index options data from 1986-1995, our tests suggest that both in- and out-of-the-money options are needed for spanning. The findings are inconsistent with deterministic volatility models but are consistent with stochastic models which incorporate additional priced risk-factors such as stochastic volatility, interest rates, or jumps. 


\section{Introduction}

What is a good model to price equity derivatives and to manage risk? Starting from Black and Scholes (1973), a common approach in the derivative pricing literature has been to model the underlying asset as a geometric Brownian motion with constant volatility. Early tests of options on stocks such as Rubinstein (1985) more or less supported the empirical implications of a geometric Brownian motion for the option market. However, after the 1987 crash, the empirical evidence is different. Using observations on S\&P500 index options from 1986 to 1992, Rubinstein (1994) documents that the violations of the Black and Scholes model are substantially larger after the 1987 crash and increasing over time. Similar patterns have also been documented in the UK, German, and Japanese index option markets by Gemmill and Kamiyama (1997). In the post-crash period, S\&P500 options exhibit a steep volatility smile, that is the implied volatility of options is often decreasing and convex in the strike price, instead of being constant as predicted by the Black and Scholes model. The size of the violations is such that they cannot be explained just by market imperfections.

Several studies have suggested extensions of the Black and Scholes model to account for the volatility smile and other empirical violations of the original model. These extensions can be loosely grouped into two main approaches: deterministic volatility models and stochastic models. Among the deterministic volatility models we find (1) the constant elasticity of variance model of Cox and Ross (1976); (2) the implied binomial tree model of Rubinstein (1994); (3) the deterministic volatility models of Dupire (1994) and Derman and Kani (1994); (4) the kernel approach by AitSahalia and Lo (1998). Among the stochastic models we find (5) the stochastic volatility models of Heston (1993), Hull and White (1987), Melino and Turnbull (1990, 1995), Scott (1987), Stein and Stein (1991), and Wiggins (1987); (6) the jump/diffusion models of Merton (1976), Bates (1991), and Madan and Chang (1996); (7) the stochastic interest rate models of Merton (1973) and Amin and Jarrow (1991).

The choice of a specific option pricing model has clearly important implications in terms of the pricing and the implementation of risk management strategies for portfolios of options and other derivatives securities. The focus of this paper is to provide a statistical test of deterministic volatility models versus stochastic models and to test if there is an additional priced risk-factor in 
the option market, such as stochastic volatility, interest rates, or jumps.

Deterministic volatility models are attractive for several reasons. First, markets are dynamically complete in these models. Thus, derivative securities can be priced by no-arbitrage without resorting to full blown general equilibrium models and without the need to estimate risk premia. Second, deterministic volatility models can potentially capture some empirical regularities, such as time-varying volatility, the correlation between volatility and the level of the underlying asset, and some forms of volatility clustering. Third, deterministic volatility models enable us to fit the smile exactly by calibrating the volatility surface of the underlying asset. The attraction here is that it is possible to generate state price densities that are consistent with current observed option prices. However, a natural concern regarding the practical use of deterministic volatility models is that the improved static pricing performance might be obtained at the cost of overfitting. In this case, the use of these models in risk management applications and for pricing exotic options would be limited.

Studies such as Bakshi, Cao, and Chen (1997), Dumas, Fleming and Whaley (1998), Bates (1998), and Jackwerth and Rubinstein (1998) have explored the empirical performance of competing option pricing models. These studies compare alternative models by measuring the models' dollar pricing and hedging errors. Jackwerth and Rubinstein (1998) run an extensive horse race including several deterministic volatility models and stochastic models. They point out that, although Black and Scholes is always outperformed by both deterministic volatility models and stochastic models, the empirical standard deviations of the pricing errors are so large that it is not possible to rank the competing models. Dumas, Fleming, and Whaley (1998) address a similar set of questions. They compare the out-of-sample performance of deterministic volatility models and characterize the trade-off between in-sample explanatory power and out-of-sample goodness of fit. Although they do not compare the performance of their deterministic volatility models to stochastic models, they show the extent of overfitting that deterministic volatility models can generate.

In this paper, we revisit the problem of which option pricing model is superior, and we introduce a formal statistical test. We do not run another horse race among a number of option pricing models based on alternative parametric specifications of the underlying asset. Instead, we construct a test of the main testable implication of any deterministic volatility model, namely, that options are redundant securities. In that case, there are no additional priced risk-factors, such as stochastic 
volatility, interest rates, or jumps. Then, the payoff of any asset can be replicated with a dynamic trading strategy, which includes two primitive assets, such as the underlying asset and the risk-free rate or, equivalently, the underlying asset and the at-the-money option. Our statistical test has the added advantage that we do not need to resort to exogenous assumptions on the shape of the potential measurement errors. The test is asymptotically consistent even when the data are measured with errors.

We ask two questions. First, do the returns on the underlying and the at-the-money option span the prices in the economy, or do we need additional information from other option returns or the risk-free rate? Second, is there any difference before and after the 1987 crash in terms of the spanning properties of the option market? We cast these questions into martingale restrictions on the pricing kernel. Testing our hypotheses within this framework has the advantage, with respect to previous empirical studies, that we can adopt a semi-parametric approach so that the empirical results are not model-specific. The only assumption that we make is that there are no arbitrage opportunities.

The only other empirical studies of the martingale restriction in option pricing are Longstaff (1995) and Brenner and Eom (1997). They provide a dollar-valued measure of the difference between the spot price and the option implied price of the underlying asset using a polynomial approximation of the pricing kernel. Longstaff (1995) tests the martingale restriction for a sample of S\&P100 American options from 1988 to 1989 using Hermite polynomials to approximate the pricing kernel. He rejects the null hypothesis that the implied level of the underlying is equal to the observed value. Brenner and Eom (1997) run a similar test using a different polynomial approximation and weekly data on S\&P500 European options from January 1993 to December 1994. They find that the implied value of the underlying in the option market is close, in economic terms, to the observed value in the spot market.

For the actual estimation of our test statistics we use two metrics. The first is the Generalized Method of Moments (GMM) metric, which is known to be optimal in the sense of attaining the asymptotic efficiency bound. A well-known problem with the GMM metric is that the optimal weighting matrix is model-dependent and asymptotic chi-square tests typically reward models that generate highly volatile pricing errors. For this reason, we also present results based on the $\delta$ metric of Hansen and Jagannathan (1997) which has the advantage of being model-independent. 
For each metric, we run conditional and unconditional tests based on the Principle of No Arbitrage, which requires that assets with the same payoff have the same price and that the state prices are non-negative in every state.

When we run the tests unconditionally, we find that both tests strongly reject the null hypothesis that in- and out-of-the-money options are redundant securities when the at-the-money option and the underlying asset are used to span the payoff space. The rejection is generally sharper post-crash. The conditional tests confirm these findings. The results reject generalizations of the Black and Scholes (1973) model based on deterministic volatility models as in the models (1)-(4) above. However, they support the alternative stochastic models where options are not redundant securities. The stochastic models (5)-(7) are characterized by their additional priced risk-factors such as stochastic volatility, interest rates, or jumps.

The structure of the paper is as follows. In section 2, we link the option returns to the pricing kernel for the economy and describe the unconditional tests based on the GMM metric. Section 3 describes the data set. Section 4 discusses the conditional tests based on the GMM metric. Section 5 investigates the small sample properties of the tests and the robustness with respect to the presence of measurement errors in option prices. The tests based on the model-independent $\delta$-metric are discussed in Section 6. Section 7 concludes.

\section{The Martingale Representation and the Cross Section of Option Prices}

In this section, we describe the methodology used to test deterministic volatility models. All noarbitrage pricing models derive the theoretical prices of contingent claims by requiring their prices to be equal to the cost of a replicating portfolio constructed from a set of securities that are presumed to span the state space. For example, any option can be replicated in the Black-Scholes economy by a portfolio consisting of the underlying asset and the bond. In the absence of transaction costs and other market frictions, this restriction is equivalent to a martingale representation of option prices. This martingale restriction is the main source of testable empirical implications, and it will be the base of our empirical analysis.

Cox and Ross (1976) develop a theory of option pricing by linking dynamic replication and 
risk neutral valuation. Harrison and Kreps (1979) and Hansen and Richard (1987) generalize this approach to a very general class of economies with the minimum requirement that securities have payoffs with finite second moments. We follow their set-up and consider a frictionless economy endowed with an objective probability space $\{\mathcal{I}, B, Q\}$. In this economy there are securities with square integrable payoffs $b_{i} \in L^{2}$ and non-singular second-moment matrix $E\left(b b^{\prime} \mid \mathcal{I}\right)$. In absence of market frictions, the space of attainable payoffs $\mathcal{P}$ is a linear space obtained as linear combinations with portfolio weights $c_{i}$ of the basis $\left\{b_{i}\right\}$ :

$$
\mathcal{P} \equiv\left\{p \in L^{2}: p=\sum_{i} b_{i} \cdot c_{i}, \text { for some } c_{i} \text { in the information set } \mathcal{I}\right\}
$$

The $c_{i}$ need not be constant and can in general be time-varying functions of the information set $\mathcal{I}$. Let $\pi(p)$ be the price of a portfolio with payoff $p$. Harrison and Kreps (1979), Chamberlain and Rothschild (1983), and Hansen and Richard (1987) show that in order for the Law of One Price to hold, the price functional $\pi(\cdot)$ must be linear ${ }^{1}$ and continuous in $p$. Since derivative securities include payoffs $h$ that are non-linear in the basis $b_{i}$ we would like to study the empirical properties of asset pricing models that extend $\pi(\cdot)$ from $\mathcal{P}$ to all of $L^{2}$. The weakest requirement for any such derivative pricing model is to satisfy the Principle of No Arbitrage.

Definition (Principle of No Arbitrage). A pricing model $\pi(\cdot)$ does not admit arbitrage opportunities in $L^{2}$ if, for any $h \in L^{2}$ such that $h \geq 0$, and $E h^{2}>0$, it is the case that $\pi(h)>0$.

Harrison and Kreps (1987) and Hansen and Richard (1987) explore the testable restrictions induced by the Principle of No Arbitrage. They show that the absence of arbitrage (a) implies the Law of One Price and (b) is equivalent to the existence of a positive pricing kernel $m>0$ such that scaled payoffs are martingales under the objective probability measure $Q$ :

Proposition (Hansen and Richard, 1987). The Principle of No Arbitrage is satisfied if and only if there exists a positive pricing kernel $m$ such that the price of any square integrable derivative security admits the following representation under the objective probability measure $Q$ :

$$
\pi_{m}(h)=E_{Q}(m h \mid \mathcal{I}), \quad \text { with } m>0 \quad \text { almost surely }
$$




\subsection{Example 1: the lognormal process}

An example of a simple economy in which the pricing kernel $m$ has well known properties is given by Black and Scholes (1973). They discuss a dynamically complete economy in which it is possible to replicate and price not only any linear portfolio in $P$ but also non-linear contingent claims, such as European options. The replicating portfolio uses dynamic trading strategies applied to a basis of $P$, consisting of the underlying asset and the risk-free rate. In this economy, the exogenous dynamics of the underlying asset are assumed to be:

$$
d S_{t}=\mu S_{t} d t+\sigma S_{t} d W_{t}
$$

Then, there exists a unique process $\xi_{T}>0$ such that: (a) $\xi_{0}=1$, (b) $\xi_{t}=E_{t}\left(\xi_{t+\Delta t}\right)$ and such that (c) $\xi_{t} S_{t}$ is a martingale. This process is given by:

$$
\begin{aligned}
\xi_{T} & =\exp \left[-\frac{1}{2} \int_{0}^{T}\left(\frac{\mu}{\sigma}\right)^{2} d t-\int_{0}^{T}\left(\frac{\mu}{\sigma}\right) d W_{t}\right] \\
& =\exp \left[-\frac{1}{2} \frac{\mu^{2}}{\sigma^{2}} T-\frac{\mu}{\sigma} W_{T}\right]
\end{aligned}
$$

so that $\xi_{T}$ is lognormally distributed with mean zero and variance equal to $\frac{\mu^{2}}{\sigma^{2}}$. The price of a call option with strike price $K$, expressed in terms of the objective probability measure, is $C_{0}=$ $E_{Q}\left[m_{0, T} \cdot \max \left(0, S_{T}-K\right) \mid \mathcal{I}_{0}\right]$ with $m_{0, T} \equiv \frac{\xi_{T}}{\xi_{0}}$ and with the solution given by the Black-Scholes formula. We note that the logarithm of the pricing kernel $m_{t, t+\Delta t}$ is exactly spanned by the $\log$ return of a portfolio formed by two assets. When these two assets are the underlying asset and a cash account $B_{t}$, with $B_{t+\Delta t}=e^{r \Delta t} B_{t}$, then the kernel-replicating portfolio is

$$
\begin{aligned}
\ln \left(m_{t, t+\Delta t}\right) & =\underbrace{\frac{1}{2} \frac{\mu}{r \sigma^{2}}\left(\mu-\sigma^{2}\right)}_{\beta_{1}} \underbrace{\ln \left(\frac{B_{t+\Delta t}}{B_{t}}\right)}_{\text {Return Asset } 1}+\underbrace{\frac{-\mu}{\sigma^{2}}}_{\beta_{2}} \underbrace{\ln \left(\frac{S_{t+\Delta t}}{S_{t}}\right)}_{\text {Return Asset } 2} \\
& =\beta^{\prime} R_{t+\Delta t}^{*}
\end{aligned}
$$

with $R_{t+\Delta t}^{*}$ being the two dimensional vector of log returns that constitutes the basis.

In a Black and Scholes economy the kernel-replicating portfolio has constant weights $\left[\frac{1}{2} \frac{\mu}{r \sigma^{2}}\left(\mu-\sigma^{2}\right), \frac{-\mu}{\sigma^{2}}\right]$. This property is not shared by the option-replicating portfolio where the delta of the option is a function of the level of the underlying. It follows that returns on options with any strike price in a Black and Scholes economy must satisfy the following testable restriction, under the objective probability measure:

$$
1=E_{Q}\left[e^{\beta^{\prime} R_{t+\Delta t}^{*}} R_{t+\Delta t} \mid \mathcal{I}_{t}\right]
$$




\subsection{Example 2: the deterministic volatility model}

Even when volatility is not constant, the pricing kernel is uniquely spanned by a portfolio of two assets as long as the volatility is deterministic. In these deterministic volatility models the price $S_{t}$ of the underlying asset is assumed to follow a generalized geometric Brownian motion

$$
\frac{d S_{t}}{S_{t}}=\mu\left(S_{t}, \mathcal{H}_{t}, t\right) d t+\sigma\left(S_{t}, \mathcal{H}_{t}, t\right) d W_{t}
$$

with $\mu(\cdot)$ and $\sigma(\cdot)$ depending on the current price of the underlying asset, time, and the history $\mathcal{H}_{t}=\left\{S_{t-h}, h>0\right\}$ of the underlying asset up to time $t$. In this class of models the pricing kernel $\xi_{T}$ is uniquely given by:

$$
\xi_{T}=\exp \left[-\frac{1}{2} \int_{0}^{T}\left(\frac{\mu_{t}}{\sigma_{t}}\right)^{2} d t-\int_{0}^{T}\left(\frac{\mu_{t}}{\sigma_{t}}\right) d W_{t}\right]
$$

$\xi_{T}$ has the property that, under the objective probability, the scaled process $\xi_{T} S_{T}$ is a martingale ${ }^{2}$ with respect to $\mathcal{I}_{t}$. Any well-behaved non-linear contingent claim with terminal payoff $p_{T}=$ $p\left(S_{T}, \mathcal{H}_{T}, T\right)$ can be priced by calculating the expected value of $\xi_{T} p_{T}$ under the objective probability measure. The price of a call option with terminal payoff $\max \left(0, S_{T}-K\right)$ is therefore $C_{0}=E_{Q}\left[m_{0, T}\right.$ $\left.\cdot \max \left(0, S_{T}-K\right) \mid \mathcal{I}_{0}\right]$ with $m_{0, T} \equiv \frac{\xi_{T}}{\xi_{0}}$. Since there are no priced risk-factors, such as stochastic volatility, interest rates, or jumps, in the deterministic volatility model the logarithm of $m_{t, t+\Delta t}$ can always be replicated by the log returns of a portfolio formed by two assets. However, as the deterministic volatility is time-varying the portfolio weights are no longer constant but are functions of the information set $\mathcal{I}_{\tau}$ :

$$
\ln m_{t, t+\Delta t}=\int_{t}^{t+\Delta t} \beta_{\tau}^{\prime} R_{\tau}^{*} d \tau
$$

with $R_{\tau}^{*}$ being the two dimensional vector of log-returns that constitutes the basis, such as the underlying asset and the risk-free asset. This implies that, in a deterministic volatility model, option returns must satisfy the following testable restriction, expressed under the objective probability measure:

$$
1=E_{Q}\left[e^{\int_{t}^{t+\Delta t} \beta_{\tau}^{\prime} R_{\tau}^{*} d \tau} R_{t+\Delta t} \mid \mathcal{I}_{t}\right]
$$

Under this hypothesis, given observations on the underlying asset and an at-the-money option, any information on other securities, such as away-from-the-money options, is redundant and not needed in order to span the pricing kernel. The advantage of considering this specification for the 
basis of the payoff space, as opposed to the more common choice of the underlying asset and the riskfree asset, is that it is independent of the observability of the risk-free asset by the econometrician. Alternatively, if stochastic volatility, interest rates, or jumps were priced in equilibrium then the pricing kernel would also be a function of any such additional risk-factor. Then, our above testable restriction fails to hold when $\beta$ is restricted to be two-dimensional as additional securities are needed for spanning. This observation constitutes the basis of our statistical test. We focus on one-period ahead pricing errors for all securities and measure the additional pricing error generated by assuming that volatility is deterministic. Equivalently, we measure the additional pricing error of assuming that just two assets span the pricing kernel.

We now turn to describe the construction of the test statistics. We partition the set of securities under investigation into two subsets $R^{*}=\left[R_{a}^{*}, R_{b}^{*}\right] . \quad R_{a}^{*}$ is the payoff space generated by the securities believed to span the entire investment opportunity set (e.g. the underlying asset and the at-the-money option), and $R_{b}^{*}$ is the payoff space of all remaining (redundant) securities, such as away-from-the-money options and the risk-free rate. If $R^{*}(\tau)$ is the instantaneous logarithmic return on a security, then

$$
\ln m_{t, t+\Delta t}=\int_{t}^{t+\Delta t} \beta_{a}^{\prime}(\tau) R_{a}^{*}(\tau)+\beta_{b}^{\prime}(\tau) R_{b}^{*}(\tau) d \tau
$$

in which the parameters $\beta(\tau)$ of the trading strategy are function of the information set $I_{\tau}$ and are approximated by a Taylor series expansion in the underlying asset. This parametrization of the pricing kernel has several desirable properties. First, since $m_{t, t+\Delta t}$ is an exponential of a linear combination of $\log$ returns it satisfies the no-arbitrage condition that $m \geq 0$ in any state of the world. Second, the payoffs $R_{t, t+\Delta t}$ which are used to identify the discount factor always contain the unit payoff. Therefore, the expected value of $m$ is equal to the value of a discount account: $E_{Q}\left[m_{t, t+1} \mid \mathcal{I}_{t}\right]=Z_{t, t+\Delta t}$. Third, since we are concerned about the possibility of rejecting the null hypothesis of spanning due to market microstructure issues, such as staleness of prices in the cash and option markets, we deliberately omit the risk-free asset from $R_{a}^{*}$, so that, in the second version of the test, $R_{a}^{*}$ contains elements of both the cash market (the underlying asset) and of the option market (the at-the-money option). We test the additional spanning provided by the away-from-the-money options.

Finally, testing if options are redundant securities is equivalent to testing, in the Euler restriction in Equation (9), that the null hypothesis $H_{o}: \beta_{b}^{\prime}(\tau)=0$ holds. The intuition of the test statistic is 
simple: under the null hypothesis that there are no additional priced risk-factors, such as stochastic volatility, interest rates, or jumps, option returns scaled by $m_{t, t+\Delta t}$ are martingales, and the sample average of $\left(m_{t, t+\Delta t} R_{t+\Delta t}-1\right)$ needs to converge to zero. Alternatively, if there are additional priced risks then $m_{t, t+\Delta t}$ cannot be spanned by only two assets and the sample average of the scaled option returns minus one converges away from zero.

Additionally, the statistical test has the following appealing economic interpretation. The expected value of $m_{t, t+\Delta t} R_{t+\Delta t}$ is the risk adjusted value of a one dollar position in an asset (or portfolio) with payoff $R$. Thus, $E_{Q}\left(m_{t, t+\Delta t} R_{t+\Delta t}-1\right)$ measures today's value of a trading strategy where we borrow one dollar to invest in a trading strategy with payoff $R_{t+\Delta t}$. Given any no-arbitrage model for $m_{t, t+\Delta t}$, the value of this portfolio should be zero if its payoff could be replicated using the same basis assets which are suggested by the model. If the null hypothesis is true and away-from-the-money options are redundant then the pricing errors should not increase when these redundant assets are not used to span the pricing kernel. As constructed, our test goes beyond testing deterministic volatility models versus stochastic models. It tests also if any additional risk-factor, such as stochastic volatility, interest rates, or jumps, is priced in equilibrium.

\section{Data}

The empirical tests are based on the Berkeley database containing all minute by minute European option quotes and trades on the S\&P500 index traded on the CBOE from April 2, 1986 to December 29, 1995. The database also contains all futures trades and quotes on the S\&P500. Our goal is to obtain a panel of daily return observations on the index, the risk-free rate, and on several options with different strike price/index level ratios (moneyness) and constant maturity.

It is important to use options with constant moneyness and maturity since our test statistics involve the conditional covariance matrix of option pricing errors. If the maturity and moneyness of options were not constant over time, then the conditional covariance matrix of the pricing errors would be time-varying, too. This would require additional exogenous assumptions on the structure of the covariance matrix and the estimation of several additional parameters, which could lead to additional estimation error in our test statistics.

- Index Level. Traders typically use the index futures market rather than the cash market to 
hedge their option positions. The reason is that the cash market prices lag futures prices by a few minutes due to lags in reporting transactions of the constituent stocks in the index. We check this claim by regressing the index on each of the first twenty minute lags of the futures price. The single regression with the highest adjusted $R^{2}$ was assumed to indicate the lag for a given day. The median lag of the index over the 1542 days from 1986 to 1992 was seven minutes. Because the index is stale, we compute a future-based index for each minute from the future market $S_{t}=\left(\frac{r_{f}}{d}\right)^{-\Delta} F_{t+\Delta}$. For each day, we use the median interest rate implied by all futures quotes and trades and the index level at that time. We approximate the dividend yield by assuming that the dividend amount and timing expected by the market were identical to the dividends actually paid on the S\&P500 index. However, some limited tests indicate that the choice of the index does not seem to affect the results of this paper.

- Interest Rates. We compute implied interest rates embedded in the European put-call parity relation. Armed with option quotes, we calculate separate lending and borrowing interest returns from put-call parity where we used the above future-based index. We assign, for each expiration date, a single lending and borrowing rate to each day which is the median of all daily observations across all striking prices. We then use the average of those two interest rates as our daily spot rate for the particular time-to-expiration. Finally, we obtain the interpolated 45 day interest rate from the implied forward curve. If there is data missing, we assume that the spot rate curve can be extrapolated horizontally for the shorter and longer times-to-expiration. Again, some limited tests indicate that the results are not affected by the exact choice of the 45 day interest rate. While we need the 45 day interest rate in order to compute the option returns, we also are interested in daily return observation of the risk-free rate. Thus, we also incorporate the federal funds (fed-funds) rate into our data-set.

- Options with Constant Moneyness and Maturity. In our data-set, all puts are translated into calls using European put-call parity. Then, we compute daily rates of return on constant maturity 45 day options with moneyness of $0.96,0.98,1,1.02$ and 1.04 . We choose 45 days as our target maturity, since, given the frequency at which new options are introduced by the exchange, every 30 days we observe options with exactly 45 days-to-expiration. In comparison, for 6 months options this happens only every 3 months. Moreover, most of the trading activity 
in the option market is concentrated in the nearest (0 to 30 days-to-expiration) and second nearest contract (30 to 60 days-to-expiration).

We choose $4 \%$ in- and out-of-the-money as our furthest moneyness levels since for short maturity options the measurement errors contained in very-deep-out-the-money options, say $10 \%$, are potentially very large as the price is relatively small with respect to the tick size.

There will normally be four observed options straddling the target moneyness and maturity. One potential way of obtaining our target option would be to use a bilinear interpolation of the implied volatility surface, as described in Fleming, Ostdiek, and Whaley (1995) and Gruenbichler and Longstaff (1996) and used for the Market Volatility Index (VIX). However, from the perspective of this paper, such interpolated option is not a traded security. Thus, it should not expected to satisfy the martingale restriction. For this reason, we use a different approach that is less flexible but more rigorous with respect to the purpose of our empirical analysis. Each trading day, we calculate the Greeks, namely delta, gamma, theta, and vega of the target (interpolated) option and the four neighboring options. Then, we find the portfolio consisting of the four market traded neighboring options which best matches the Greeks of the target option while limiting the weights to the interval 0 to 1 . The portfolio weights are then kept fixed and the value of this tradable portfolio is marked-to-market the following day using available market quotes. The advantage of this approach is that, since this portfolio is a traded asset, then its return must be a martingale under the risk neutral probability measure. This fact holds even if the weights are incorrect because we misspecified the reference model that is used to compute the Greeks.

- Arbitrage Violations. In the process of setting up the database, we check for a number of errors which might have been contained in the original minute by minute transaction level data. We eliminate a few obvious data-entry errors as well as a few quotes with excessive spreads more than 200 cents for options and 20 cents for futures. General arbitrage violations are eliminated from the data-set. Within each minute we keep the largest set of option quotes which does not violate:

$$
S d^{-t} \geq C_{i} \geq \max \left[0, S d^{-t}-K_{i} r_{f}^{-t}\right]
$$

We also check for violations of vertical and butterfly spreads. 


\section{TABLE 1, about here}

We restrict our attention on options which are no more than $4 \%$ away-from-the-money. The reason is due to the depth of the market and to the relative impact of measurement errors for options which are deep out-the-money. Table 1 presents the average daily notional volumes of traded S\&P500 index options for the moneyness levels used in the empirical analysis. The volumes are aggregate numbers across the two contracts with maturities straddling 45 days. The total market value of at-the-money options has increased ten-fold over 10 years. In 1995, the aggregate volume of at-the-money options was $\$ 263 \mathrm{~m}$. For moneyness levels out to $\pm 5 \%$, the CBOE option market is very active. In 1995, the volume of options with moneyness levels between 0.95 and 0.97 was $\$ 119 \mathrm{~m}$, still almost half of the at-the-money volume. The volume of options with moneyness levels between 1.03 and 1.05 was $\$ 63 \mathrm{~m}$. However, beyond moneyness levels of 1.05 the option volumes are substantially lower.

Tables $2_{a}$ and $2_{b}$, about here

Tables $2_{a}$ and $2_{b}$ report sample statistics of the data-set which consists of 2426 observations for each of our seven daily return series. These seven series are the returns of the underlying asset, the risk-free rate for which use the fed-funds rate as a proxy, and five 45 day options with moneyness levels of $0.96,0.98,1,1.02$, and 1.04. We term these options the deep-in-the-money, in-the-money, at-the-money, out-of-the-money, and deep-out-of-the-money call options, respectively. The mean returns and volatilities of the options may appear high with respect to the return and volatility of the market. This is due to the leverage implicit in option positions. Option volatilities and excess returns over short horizons such as the present one day are multiples of the market return, with the multiplier being the elasticity of the option price with respect to the index level $\left(=\Delta \frac{S}{C}\right)$. As a rough check, we use the Black-Scholes formula to calculate options prices and deltas based on the historical sample averages for the interest rate of $5.43 \%$, the dividend yield of $3.12 \%$, and the volatility of $16.16 \%$. The index level is set to 1 without loss of generality and the strike prices are $0.96,0.98,1.00,1.02$, and 1.04. Time-to-maturity is 45 days. The elasticities for the five options are $16,19,22,26$, and 30 . That would imply returns on these options of 82, 97, 112, 132, and 152 
basis points per trading day and volatilities of 1376, 1634, 1892, 2236 and 2580 basis points per trading day.

The volatilities of the observed option returns line up nicely, except for the deep-out-of-themoney option which has an observed volatility of 3431. Potential reasons for such deviation are our assumption that the Black-Scholes formula holds with the same parameters for about a decade and the fact that the deep-out-of-the-money options are the least liquid of the five options. Continuing our comparisons, the average returns of the observed options are lower than in the Black and Scholes case, except for the deep-out-of-the-money options. However, the estimates of the average returns in the Black-Scholes case have very wide $99 \%$ confidence intervals which we base on $\pm 3 \frac{\sigma}{\sqrt{T}}$ $(=74,89,111,147$, and 208). Namely, the lower limits are 8, 8, 1, -15, and -56 and the upper limits are 156, 186, 223, 279, and 360. Then, all observed option returns lie within the \pm 3 standard deviation band.

Although this may be interpreted as an indication of a risk premium implicit in option prices, it can still be reconciled with more flexible deterministic volatility models in which volatility is not restricted to be flat and constant at $16.16 \%$, as in the Black and Scholes reference case. Adjusting the option elasticities for the volatility smile, which associates higher (lower) implied volatilities with in-the-money (out-of-the-money) options, would lead to results more in line with the observed returns and volatilities.

Still in Table $2_{a}$, we find that the autocorrelation coefficients quickly decay to zero and, with the exception of the risk-free rate, are not significantly different from zero. In the following Table $2_{b}$ we report the contemporaneous correlation between the seven assets. As expected, the correlations are higher for options with more similar moneyness levels.

\subsection{The unconditional test}

A necessary condition for the Principle of No Arbitrage can be obtained by taking unconditional expectations of Equation (9), from which:

$$
1=E\left(m_{t, t+\Delta t} R_{t+\Delta t}\right)
$$

Let $R_{t, t+\Delta t, n}^{*}$ be the vector of log returns sampled at intervals ${ }^{3} \Delta t / n$ that are used to span the finite sample analog of the pricing kernel $m_{t, t+\Delta t}$ in Equation (11). In the unconditional case, we 
focus on the time-series properties of the one-period ahead unexpected returns:

$$
h_{t+\Delta t}^{\prime}\left(m\left(\beta, R^{*}\right), R\right) \equiv 1^{\prime}-m\left(\beta, R^{*}\right) R_{t, t+\Delta t}^{\prime}
$$

Under the null hypothesis that the model is correctly specified, $h_{t+\Delta t}$ is a martingale in sequence. Let the time average of the one period unexpected returns be $h_{T}=\frac{1}{T} \sum_{t=1}^{T} h_{t}$ so that, if $R_{t, t+\Delta t}$ is stationary, $h_{T} \rightarrow E\left(h_{t+\Delta t}\right)$. Hansen (1982) obtains a consistent estimators of $\beta$ by minimizing the GMM quadratic form of the average unexpected returns $h_{T}^{\prime} W_{T}^{-1} h_{T}$ :

$$
\hat{\beta} \equiv \operatorname{argmin}\left[T \cdot h_{T}^{\prime}(\beta) W_{T}^{-1} h_{T}(\beta)\right]
$$

The weighting matrix $W_{T}$ is chosen to be a consistent estimator of the unconditional asymptotic covariance of the pricing errors $h_{t}$. Such consistent estimators can be computed using the frequency zero spectral density estimators developed by Newey and West (1987) and Andrews (1991). The, Hansen (1982) shows that $\hat{\beta}$ is efficient and $\sqrt{T}\left[\hat{\beta}-\beta_{o}\right]$ is asymptotically normally distributed with

covariance matrix $\left[\left(E \frac{\partial h_{T}^{\prime}}{\partial \beta}\right) W_{T}^{-1}\left(E \frac{\partial h_{T}}{\partial \beta}\right)\right]^{-1}$. Let $h_{T}^{r}$ be the restricted counterpart of $h_{T}$, under the null hypothesis that $H_{o}: \beta_{b}=0$, then the following asymptotic result holds:

\section{Test 1 (Unconditional Test, GMM metric)}

Under the null hypothesis that the assets in $R_{b}$ are redundant given $R_{a}$,

$$
d_{T} \equiv T\left[h_{T}^{r}\left(\hat{\beta}_{a}\right)-h_{T}(\hat{\beta})\right]^{\prime} W_{T}^{-1}\left[h_{T}^{r}\left(\hat{\beta}_{a}\right)-h_{T}(\hat{\beta})\right] \rightarrow \chi_{\left(n_{b}\right)}^{2}
$$

is asymptotically distributed chi-square with $n_{b}$ degrees of freedom, where $n_{b}$ is the number of redundant securities.

The statistic is a quadratic form of the increase in the unexpected returns due to the null hypothesis that only two are needed to span the pricing kernel. If the null hypothesis is satisfied then the pricing errors should not increase significantly as the null hypothesis is imposed and $d_{T}$ should be close to zero. Other examples of such region subset tests are Braun (1992), Cochrane and Hansen (1992), DeSantis (1993), Knez (1993), and Bekaert and Urias (1996).

\subsection{Empirical results: the unconditional test}

In a first series of tests we choose the sampling frequency $\Delta t$ to be one day. However, the results are robust to changes in the sampling frequency and we report the details below. The unconditional 
test of the martingale condition in Equation (14) is based on seven pricing restrictions, with $R_{t+\Delta t}$ being the $(7 \times 1)$ vector of returns on all the available unconditional investment opportunities, that is the underlying asset, the risk-free rate, and the five options. The weighting matrix $W_{T}$ is the Newey-West (1987) heteroskedastic and autocorrelation consistent estimator of the unconditional covariance matrix of the seven unexpected returns $h_{t}$ :

$$
\begin{aligned}
W_{T} & =\Gamma_{0}+\sum_{i=1}^{q}\left(1-\frac{i}{q+1}\right)\left(\hat{\Gamma}_{i}+\hat{\Gamma}_{-i}^{\prime}\right) \\
\hat{\Gamma}_{i} & =\frac{1}{T} \sum_{t=i+1}^{T}\left(h_{t}-\bar{h}\right)\left(h_{t-i}-\bar{h}\right)^{\prime} \text { and } \bar{h}=\frac{1}{T} \sum_{t=i+1}^{T} h_{t}
\end{aligned}
$$

We choose $q$ to be five trading days (one week). In TABLE 3 we show the empirical evidence from the unconditional chi-square test of $H_{o}: \beta_{b}=0$.

TABLE 3, about here

For our tests, we specify three different sets of basis $R_{a}^{*}$ and non-basis $R_{b}^{*}$ returns. In test $I I I_{a}$, the null hypothesis states that observations on deep-in- and deep-out-of-the-money options do not add relevant information to identify the pricing kernel beyond the information already contained in the returns on the underlying asset and the risk-free rate. In the post-crash and the overall samples, we strongly reject the hypothesis that the away-from-the-money option returns are redundant at any confidence level. We find that they price additional risk-factors that are not already spanned by at-the-money options and the underlying asset. However, in the pre-crash sample, the awayfrom-the-money options are redundant at the $34 \%$ confidence level.

In test $I I I_{b}$, we change the basis assets slightly and replace the risk-free rate with the atthe-money option. This specification allows us to address concerns about market microstructure differences between the cash market and the option market. If there were a sufficiently large lag between the time at which information is priced in the market for the underlying asset and in the option market then the null hypothesis might be rejected even if the underlying asset followed a deterministic volatility model. We can ameliorate these concerns by incorporating both the underlying asset and an option into the basis set of reference assets. The results of test $I I I_{b}$ indicate that the away-from-the-money-options are now needed in all three sample periods for spanning purposes. Even in the pre-crash period do we need the away-from-the-money options, as 
opposed to the findings in $I I I_{a}$. One potential reason might be that we do not have any information on the risk-free rate available for spanning the pricing kernel.

Therefore, in test $I I I_{c}$, we investigate if the risk-free rate adds information beyond the returns on the underlying and the at-the-money option. In the post-crash period we find that the risk-free rate does add information beyond what is already contained in the two basis securities. However, the risk-free rate is redundant in the pre-crash period at the $70 \%$ confidence level.

The results of the spanning tests raise doubts on the effectiveness of using highly pliable deterministic volatility models for risk management and pricing purposes. The results of the tests suggest that there is at least one additional priced risk-factor, such as stochastic volatility, interest rates, or jumps.

\section{The Conditional Test}

The unconditional pricing restriction in Equation (11) is only a necessary condition for the Principle of No Arbitrage to hold. For this reason, we now study the null hypothesis that the non-basis returns are redundant in a conditional setting:

$$
0=E\left[h_{t+\Delta t} \mid \mathcal{I}_{t}\right]
$$

with the unexpected returns $h_{t+\Delta t}$ defined as in Equation (12). In contrast with the unconditional case, we assume that arbitrageurs may take hedging decisions conditional on non-trivial information about the state of the economy. Let $\phi\left(\omega_{t}\right)$ be an $I_{t}$-measurable function of a set of financial variables measurable at time $t$ such as the level of the underlying asset or the slope of the volatility smile. Then, we can construct managed portfolios with initial cost $\phi\left(\omega_{t}\right)$ and payoff $R_{t+\Delta t} \phi\left(\omega_{t}\right)$. Any return in the economy, such as returns on the underlying asset, the risk-free rate, the options, or any managed portfolio, has to satisfy Equation (15), that is, both $0=E\left(h_{t+\Delta t} \mid I_{t}\right)$ and $0=E\left[\phi\left(\omega_{t}\right)\right.$ $\left.\times h_{t+\Delta t} \mid I_{t}\right]$ need to be satisfied for any $I_{t}$-measurable function $\phi\left(\omega_{t}\right)$. After taking unconditional expectations we obtain the following expression:

$$
0=E\left[\begin{array}{ll}
h_{t+\Delta t} & \\
h_{t+\Delta t} & \phi\left(\omega_{t}\right)
\end{array}\right]
$$

Let $m\left(\beta_{t}, R_{t, t+\Delta t, n}^{*}\right)$ be the time-varying pricing kernel of the deterministic volatility model. The discrete and time-varying parameters $\beta_{t} \in I_{t}$ are parameterized with polynomial expansions 
in the state variable:

Black-Scholes model: $\beta(S, \tau)=\beta_{o}$

Model 1: $\beta(S, \tau)=\beta_{o}+\beta_{1} S_{\tau}$

Model 2: $\beta(S, \tau)=\beta_{o}+\beta_{1} S_{\tau}+\beta_{2} S_{\tau}^{2}$

Model 3: $\beta(S, \tau)=\beta_{o}+\beta_{1}\left(S_{\tau}-E_{\tau-1}\left(S_{\tau}\right)\right)$

Model 4: $\beta(S, \tau)=\beta_{o}+\beta_{1}\left(S_{\tau}-E_{\tau-1}\left(S_{\tau}\right)\right)+\beta_{2}\left(S_{\tau}-E_{\tau-1}\left(S_{\tau}\right)\right)^{2}$

So far, we have been using the Black-Scholes Model in our unconditional tests. Models 1 and 2 are more general specifications of deterministic volatility models along the lines of Dumas, Fleming, and Whaley (1998). Since the underlying asset is drifting over time, we consider two additional specifications, models 3 and 4, that are expressed in terms of the conditionally demeaned level of the underlying asset. Model 4 is particularly interesting since it expresses the pricing kernel in terms of the square conditional innovations of the underlying asset. Although model 4 is still deterministic and its dynamics are generated by just one Brownian motion it may capture some of the volatility clustering effects that have partly motivated the development of stochastic volatility models. Finally, we again directly parametrize the functional form of the pricing kernel:

$$
m_{t, t+\Delta t}=\exp \left[\sum_{j=0}^{n-1} \beta_{a}^{\prime}\left(S_{t_{j}}, t_{j}\right) R_{a, t_{j}, t_{j+1}}^{*}+\beta_{b}^{\prime}\left(S_{t_{j}}, t_{j}\right) R_{b, t_{j}, t_{j+1}}^{*}\right]
$$

with $t_{0}=t$ and $\sum_{j=0}^{n-1}\left[t_{j+1}-t_{j}\right]=\Delta t$.

We estimate the parameters $\beta_{t}$ using the moment condition in Equation (16) and minimizing the quadratic GMM criterion:

$$
J_{T}=\left[\frac{1}{\sqrt{T}} \sum_{t=1}^{T}\left(\begin{array}{ll}
h_{t+\Delta t} & \\
h_{t+\Delta t} & \phi\left(\omega_{t}\right)
\end{array}\right)\right]^{\prime} W_{T}^{-1}\left[\frac{1}{\sqrt{T}} \sum_{t=1}^{T}\left(\begin{array}{ll}
h_{t+\Delta t} & \\
h_{t+\Delta t} & \phi\left(\omega_{t}\right)
\end{array}\right)\right]
$$

Let the sample average of the conditional pricing errors be $h_{T} \equiv \frac{1}{T} \sum_{t=1}^{T}\left[h_{t+\Delta t}^{\prime}, \phi^{\prime}\left(\omega_{t}\right) \quad h_{t+\Delta t}^{\prime}\right]^{\prime}$, and let the superscript $r$ denote the unexpected returns of the restricted model. If $W_{T}$ is chosen to be a consistent estimator of the asymptotic covariance matrix of $\left[\begin{array}{ll}h_{t+\Delta t}^{\prime}, \phi^{\prime}\left(\omega_{t}\right) & h_{t+\Delta t}^{\prime}\end{array}\right]^{\prime}$ then the $G M M$ estimator of $\beta$ is also efficient. In this case, the following asymptotic result holds:

\section{Test 2 (Conditional Test, GMM metric)}


Under the null hypothesis that the assets in $R_{b}^{*}$ are redundant given $R_{a}^{*}$,

$$
d^{c} \equiv T\left[h_{T}^{r}\left(\hat{\beta}_{a}\right)-h_{T}(\hat{\beta})\right]^{\prime} W_{T}^{-1}\left[h_{T}^{r}\left(\hat{\beta}_{a}\right)-h_{T}(\hat{\beta})\right] \rightarrow \chi_{\left(n_{\xi} n_{b}\right)}^{2}
$$

is asymptotically distributed chi-square with $n_{\xi} \times n_{b}$ degrees of freedom, where $n_{\xi}$ and $n_{b}$ are respectively the number of conditioning variables and the number of redundant securities.

Similar to the unconditional case, we consider a Newey-West autocorrelation and heteroskedasticity consistent estimator of the covariance matrix of the pricing errors with five lags.

\subsection{Empirical results: the conditional test}

We now present the empirical results of the conditional tests which incorporate returns on timevarying managed portfolios. The pricing kernel replicating portfolio will then also have time-varying portfolio weights $\beta_{\tau}$ as described above.

\section{TABLE 4, about here}

Test $I V_{a}$ presents the results of the test of redundancy of the away-from-the-money option returns while conditioning on functions of the level of the underlying asset. Across all models, we find the unconditional results repeated and for the most part more pronounced. The p-values in the pre-crash sample tend to be even higher than in the unconditional case while they were even lower than the unconditional case in the post-crash and overall samples. Test $I V_{b}$ shows that if the at-the-money option is included in the basis set then spanning is rejected even in the pre-crash sample and more strongly so in the post-crash and overall samples. Again, the results tend to be more pronounced than in the unconditional case. Test $I V_{c}$ investigates the redundancy of the risk-free rate, and the results strengthen the findings of the unconditional test. Overall, all unconditional results are confirmed both in the specification expressed in terms of the absolute level of the underlying asset (model 1 and model 2) and in the specification expressed in terms of conditionally demeaned levels of the underlying asset (model 3 and model 4).

\section{Robustness of the Tests}

Here we discuss the sensitivity of the results to some aspects of the empirical implementation of the tests. Namely, we consider the sampling frequency of our returns, the use of additional conditioning 
variables, the dependence of our results on trading volume, and finally, the differential spanning properties of option returns with different moneyness. Thereafter, we explore the small sample properties of the tests without and with measurement errors.

So far, the sampling frequency $\frac{\Delta t}{n}$ of all returns on the seven assets was daily, that is $\Delta t=1$ day and $n=1$ day, and the results have been reported in Tables 3 and 4 . We test the robustness of our results by re-running tests based on different sampling frequencies $\frac{\Delta t}{n}$. First, we run the test statistics for $\Delta t=1$ day and $n=2$ days, that is we measure two-day returns and can rebalance once after one day. Second, we use $\Delta t=1$ day and $n=5$ days, that is we measure weekly returns and we can rebalance after every working day of the week. We find that the results closely track the ones for the original daily sampling and that the conclusions are not affected.

Next, we check the sensitivity of the conclusions to different choices for the conditioning variables used to obtain time-varying managed portfolios. In addition to polynomials in the absolute and conditionally demeaned level of the underlying asset, we also consider the slope of the smile and the level of the interest rate as conditioning variables. We find that the results closely mirror the ones in Table 4.

We also consider the dependence of our results on trading volume. For this purpose we create two subsamples based on trading volumes. In the first subsample, we consider the returns on options only on those days where the daily aggregate trading volumes in the option market are in the top three deciles of the distribution. In the second subsample, we consider the days in which volumes are in the bottom three deciles. Then, we compare the test statistics. In the post-crash period, the hypothesis of redundancy of the away-from-the-money options is rejected less strongly in the subsample with the high trading volumes. However, even for the subsample with high trading volumes the null hypothesis is soundly rejected at confidence levels below $1 \%$. In the precrash period, the cross-section of options remains redundant in both subsamples. As expected, we conclude that a relatively thin market may have an impact on the ability to replicate the options. Our findings are supported by the conditional tests and by the tests based on the $\delta$-metric.

Finally, we investigate if returns on options with different moneyness have differential spanning properties. Out-of-the-money call or put options are much more liquid than in-the-money call or put options, and put option volume is higher than call option volume by a factor of about 3 . Since we translate all put quotes into call quotes, we find as expected that the trading volume 
at low moneyness levels (which is driven by the out-of-the-money puts) is higher than for high moneyness levels (which is driven by the out-of-the-money calls). Table 1 confirms that in 1995, the average daily trading volume of options with moneyness between 0.95-0.97 was twice as high as that of options with moneyness between 1.03-1.05. Such difference in cross-sectional liquidity could be responsible for different spanning properties. We address this issue by running two sets of tests. In the first test, the basis is defined as $R_{a}^{*}=\left[R_{s}, R_{0.96}\right]$ while in the second test the basis is specified as $R_{a}^{*}=\left[R_{s}, R_{1.04}\right]$. We ask if the spanning properties of in-the-money and out-the-money options are symmetric, and our tests confirm that this is indeed the case. This suggests that the non-redundancy of the cross-section of option returns is not simply due to liquidity reasons.

\subsection{Small-sample properties}

The above empirical findings are all based on asymptotic test statistics. This might be considered adequate since the data-set contains 2426 daily observations throughout almost 10 years. Nonetheless, since the previous tests sharply reject the null hypothesis that there are no priced additional risk-factors, we want to check the robustness of the tests. In our first simulation study, we study the performance of the test statistics in small samples and the approximation error generated by discretizing the continuous time pricing kernel. In our second simulation study, we add measurement error, too.

In order to see if the test rejects the null hypothesis even when the null hypothesis is true, we simulate 1000 days of a cross-section of option prices under the assumption that the underlying asset follows a constant elasticity of variance (CEV) process. The CEV model is a deterministic volatility model and only two basis returns are needed for spanning. We then run the spanning tests and report the results in Table 5.

\section{TABLE 5, about here}

Given a theoretical p-value of $5 \%$, we measure the empirical frequency that the test rejects the null hypothesis, that is the simulated $d_{T}$ is higher than the rejection level implied by the chi-square distribution for a p-value of $5 \%$. The difference between the empirical rejection frequency and the $5 \%$ value is a measure of over-rejection. If there is no bias, this value should asymptotically be equal to zero. When measurement errors are zero, we find that the empirical rejection frequency is 
very close to the theoretical level: we reject the null hypothesis $3.45 \%$ and $4.02 \%$ of times in test A and test B, respectively, and we find a slight under-rejection.

We also consider the approximation error generated by discretizing the continuous time pricing kernel since we simulate the CEV process with 100 intermediate steps within each day. However, we only observe daily option returns and can only trade once a day. Still, our simulated rejection frequencies are very close to the theoretical $5 \%$ level.

\subsection{Small-sample properties with measurement errors}

For our second simulation study we consider the case in which option prices are observed with errors. Let $R_{t}$ be the return on option prices simulated in a CEV environment and let $\hat{R}_{t}$ be the observed option return, with $\hat{R}_{t}=R_{t}+\sigma_{\varepsilon} \varepsilon_{t}$. Given a value for $\sigma_{\varepsilon}$, the standard deviation of the measurement errors, we estimate the empirical frequency of rejection of the null hypothesis that options are generated by a deterministic volatility model. In order to run the simulation, we need a reasonable value for $\sigma_{\varepsilon}$. We adopt an extension of the model of Roll (1984) and assume that the option excess returns $R_{t+1}$ adhere to the following autoregressive process:

$$
R_{t+1}=\mu+\rho R_{t}+\sigma_{\eta} \eta_{t+1} ; \eta_{t+1} \sim \text { i.i.d. }(0,1)
$$

If returns are observed with error $\varepsilon_{t+1}$ then:

$$
\hat{R}_{t+1}=R_{t+1}+\sigma_{\varepsilon} \varepsilon_{t+1} ; \quad \varepsilon_{t+1} \sim \text { i.i.d. }(0,1)
$$

Since measurement errors appear both on the left and right hand side of the equation, we can identify the sampling errors from the innovations of the true process. Straightforward calculations show that, given equations (19) and (20), the unconditional population moments of the observed returns satisfy the following restrictions:

$$
\begin{aligned}
E_{0}\left[\widehat{R}_{t}-\frac{\mu}{1-\rho}\right] & =0 \\
E_{0}\left[\widehat{R}_{t-1}\left(\widehat{R}_{t}-\mu-\rho \widehat{R}_{t-1}\right)\right] & =0 \\
\operatorname{Var}_{0}\left[\Delta \widehat{R}_{t}\right] & =2 \sigma_{\varepsilon}^{2}+\sigma_{\eta}^{2}\left(\frac{2}{1+\rho}\right) \\
\operatorname{Cov}_{0}\left[\Delta \widehat{R}_{t}, \Delta \widehat{R}_{t-1}\right] & =-\sigma_{\varepsilon}^{2}-\sigma_{\eta}^{2}\left(\frac{1-\rho}{1+\rho}\right)
\end{aligned}
$$




$$
\operatorname{Cov}_{0}\left[\Delta \widehat{R}_{t}, \Delta \widehat{R}_{t-2}\right]=\rho(1-\rho) \frac{1+2 \rho}{1+\rho} \sigma_{\eta}^{2}
$$

The model is overidentified and we can estimate the four parameters using the five moment

restrictions. We find that the noise to signal ratio in the data-set is $\frac{\sigma_{\eta}}{\sigma_{\varepsilon}}=1.74 \%$ with $\sigma_{\varepsilon}=38$ basis points. This compares with an average bid/ask spread of 83 cents for the at-the-money-option. We conclude that there is sampling error, thus the need for a statistical test, but the size compares very favorably with daily bid-ask spreads. We then simulate the returns and conduct the spanning tests. For safety, we use a value for $\sigma_{\varepsilon}$ of 600 basis points which roughly matches the size of the bid/ask spread. Table 6 reports the results.

TABLE 6, about here

Even with such a large value for the measurement error, we find that the empirical rejection frequency is only $0.36 \%$ higher than the theoretical value when $\rho=\frac{1}{2}$. For test A, the highest level of small sample bias is found in the case of $\rho=1$. In this case, we reject the null hypothesis $1.63 \%$ more frequently than the $5 \%$ value implied by the asymptotic distribution. The size of the small sample bias is too small to explain the sharp rejections found when the test is run on actually observed option returns. It should be noticed that in order to better capture the small sample bias, we simulate time series of option prices for 1000 trading days, less than half the size of the data-set that we actually have. Therefore, the average small sample bias should be even smaller when we run the empirical test on 2426 observations. We conclude that the rejection of the null hypothesis based on empirical option returns is unlikely to be simply due to the presence of measurement error. This seems reasonable since the measurement errors induce a bias in the test statistics only if they are also priced.

\section{$5 \quad$ Tests Based on the Model-Independent $\delta$-Metric}

Using both an unconditional and conditional approach, the tests based on the asymptotically efficient GMM metric strongly reject the null hypothesis that there are no additional priced riskfactors. We are concerned that our results could be driven by the particular GMM metric used to weight the pricing errors. In any GMM metric, the pricing errors are weighted by an estimate of the inverse of the asymptotic covariance matrix. However, a well-known undesirable feature of the 
GMM metric is that it rewards models characterized by pricing kernels with large variances. The larger the variance of the pricing kernel, the larger the variance of the pricing errors and therefore the smaller the weighted quadratic criterion, for any given average level of pricing error. Moreover, the GMM test need not be optimal in small samples.

Thus, in order to evaluate the robustness of the GMM results, we consider the alternative approach by Hansen and Jagannathan (1997), which is not subject to the previous criticism. Let $m(\beta)$ be a candidate parametric pricing kernel that is used to generate approximate prices for securities with payoff $p \in \mathcal{P}$, so that $\pi_{m(\beta)}(p)=E(m(\beta) p)$. In deterministic volatility models, a candidate pricing kernel $m(\beta)$ can be written as an exponential function of the continuously compounded log return of a portfolio of two spanning assets. Since $m(\beta)$ completely describes the asset pricing implications of any candidate model, a ranking of alternative candidate models can be developed in terms of the pricing errors that are generated by different candidate pricing factors $m(\beta)$. Since any measure of pricing performance of a model depends on the specific assets used as benchmarks, Hansen and Jagannathan (1997) explore the properties of the maximum pricing error generated by the candidate pricing kernel $m(\beta)$ with respect to all portfolios with payoff $p$. Since any pricing deviation can be made arbitrarily large by increasing the leverage of the portfolio, they consider the payoffs with unit second moment, $E\left(p^{2}\right)=1$, and define the following measure of pricing performance:

$$
\delta \equiv \max _{p \in \mathcal{P}, E\left(p^{2}\right)=1}\left|\pi_{m(\beta)}(p)-\pi(p)\right|
$$

where $\pi(p)$ are observed prices of the securities with payoff $p$. Thus, a model $m(\beta)$ is said to be superior to an alternative model $m\left(\beta^{\prime}\right)$ if the maximum pricing error, in absolute value, is lower than the one generated by $m\left(\beta^{\prime}\right)$. Clearly, this metric of model performance does not require the two models to be nested. Hansen and Jagannathan (1997) show that a bound on the maximum pricing error is given by the smallest second moment distance between the candidate pricing kernel $m(\beta)$ and the space ${ }^{4}$ of admissible pricing kernels $\tilde{m} \in M$ :

$$
\delta=\min _{\tilde{m} \in \mathcal{M}} E(m(\beta)-\tilde{m})^{2}
$$

The programming problem in Equation (25) is defined with respect to an infinite dimensional control variable. By considering its dual representation Hansen and Jagannathan (1997) show that 
its solution is given by:

$$
\delta^{2}=[\operatorname{Epm}(\beta)-E \pi(p)]^{\prime} E p p^{\prime}[\operatorname{Epm}(\beta)-E \pi(p)]
$$

The measure of pricing performance differs from the usual GMM metric since it depends on a weighting matrix $E p p^{\prime}$, which is invariant to the choice of the pricing kernel. There are two advantages to the $\delta$-metric. First, it is directly related to an economic concept, namely, the maximum pricing error among all payoffs with standardized second moments. Second, it is invariant to the volatility of the pricing imprecision and, more generally, to the statistical properties of the model that is being tested.

However, since the $\delta$-metric is not based on the asymptotically optimal weighting matrix, the asymptotic distribution of the sample counterpart of $\delta$ is not chi-square. In order to describe the spanning test defined in terms of the $\delta$-metric, let $Q_{T}$ be a consistent estimator of the second moment matrix of the unit cost payoffs (returns) $Q_{T} \rightarrow Q \equiv E\left(R_{t}^{\prime} R_{t}\right)$ and similarly to previous sections, let $h_{T}$ be the sample average of the pricing errors of unit cost payoffs, $h_{T}=\frac{1}{T} \sum_{t=1}^{T}\left(1-R_{t} m_{t}(\beta)\right)$. Consistent estimators of $\beta$ can be obtained by minimizing the following sample analog of the $\delta$ metric:

$$
d_{T}^{2}(\beta)=h_{T}(\beta)^{\prime} Q_{T}^{-1} h_{T}(\beta)
$$

Using results in Jagannathan and Wang (1997) we show in the appendix that:

\section{Test 3 (Unconditional Test, $\delta$-metric)}

Under the null hypothesis that the assets in $R_{b}$ are redundant given $R_{a}$ and the unrestricted model is overidentified, $T\left[d_{T}^{2}\left(\hat{\beta}_{r}\right)-d_{T}^{2}(\hat{\beta})\right]$ converges in distribution to a weighted sum of $n_{b}$ chi-square distributions with one degree of freedom $\sum_{i=1}^{n_{b}} \lambda_{i} \chi_{(1)}^{2}$.

The values of $\lambda_{i}$ are the positive eigenvalues of a transformation of the score of the pricing errors and they are fully characterized in the Appendix. The distribution can be tabulated numerically. When the unrestricted model is exactly identified then $d_{T}^{2}(\hat{\beta})$ is identically equal to zero. However, the unrestricted model is never exactly identified in our implementations.

Next, we turn to the conditional test based on the $\delta$-metric. The restriction $E(m R-1)=0$ is only a necessary condition for $E\left(m R-1 \mid \mathcal{I}_{t}\right)$ to hold. Therefore, we explore a conditional version of the test and we use the $\delta$-metric to construct a spanning test valid also for time-varying managed 
portfolios with initial cost $\phi\left(\omega_{t}\right)$ and following period payoff $R_{t+\Delta t} \phi\left(\omega_{t}\right)$. Let $\phi\left(\omega_{t}\right)$ be a function measurable with respect to the information set $\mathcal{I}_{t}$. Multiplying the conditional moment restriction by $\phi\left(\omega_{t}\right)$ and taking unconditional expectations, the conditional counterpart of Equation (27) is given by the following criterion:

$$
d_{T}^{2}(\beta)=\left[\frac{1}{\sqrt{T}} \sum_{t=1}^{T}\left(\begin{array}{cc}
h_{t+\Delta t} & \\
\phi\left(\omega_{t}\right) & h_{t+\Delta t}
\end{array}\right)\right]^{\prime} Q_{T}^{-1}\left[\frac{1}{\sqrt{T}} \sum_{t=1}^{T}\left(\begin{array}{cc}
h_{t+\Delta t} & \\
\phi\left(\omega_{t}\right) & h_{t+\Delta t}
\end{array}\right)\right]
$$

with $Q_{T}$ being a consistent estimator of $E_{t}\left[\left(\begin{array}{cc}R_{t+1} & \\ R_{t+1} & \phi\left(\omega_{t}\right)\end{array}\right)\left(\begin{array}{cc}R_{t+1} & \\ R_{t+1} & \phi\left(\omega_{t}\right)\end{array}\right)^{\prime}\right]$.

As usual, the weights of the time-varying kernel-replicating portfolio are parametrized as in Equation (17). This should capture more complex forms of non-linearities of the pricing kernel, beyond the exponentially linear case.

\section{Test 4 (Conditional Test, $\delta$-metric)}

Under the null hypothesis that the assets in $R_{b}$ are redundant given $R_{a}$ and the unrestricted model is overidentified, $T\left[d_{T}^{2}\left(\hat{\beta}_{r}\right)-d_{T}^{2}(\hat{\beta})\right]$ converges in distribution to a weighted sum of $n_{\xi} \cdot n_{b}$ chi-square distributions with one degree of freedom $\sum_{i=1}^{n_{\xi} n_{b}} \lambda_{i} \chi_{(1)}^{2}$, where $n_{\xi}$ is the number of conditioning variables defining the dynamic trading strategy $\xi\left(\omega_{t}\right)$.

\subsection{Empirical results based on the model-independent $\delta$-metric}

We report the results for the unconditional tests based on the $\delta$-metric in Table 7.

\section{TABLE 7, about here}

When we compare the results to the GMM results in Table 3, we find them to be very similar for tests $V I I_{a}$ and $V I I_{b}$. These two tests consider the incremental spanning due to the away-fromthe-money options. Interestingly, the results for the incremental spanning due to the risk-free rate, which are reported in $V I I_{c}$, did not change either, but the p-values increased in the post-crash and overall samples. This pattern is even more pronounced in the conditional tests which we report in Table 8.

TABLE 8, about here 
We compare the results to the conditional GMM results in Table 4. As above, the results are virtually identical for tests $V I I I_{a}$ and $V I I I_{b}$ where we consider the incremental spanning due to the away-from-the-money options. Again, we observe that the conditional tests based on the $\delta$-metric do not confirm the GMM results that the risk-free rate is needed for spanning in the post-crash and overall samples. According to test $V I I I_{c}$ which reports the results under the $\delta$-metric, the conditional tests for model 4 do not reject the null hypothesis of redundancy of the risk-free rate in any subsample. Additionally, for model 3 in the overall sample we cannot reject the null hypothesis of redundancy of the risk-free rate at the $27 \%$ confidence level. This evidence is of particular methodological interest as it shows the importance of comparing tests based on the GMM metric to tests based on the $\delta$-metric. It also suggests that additional priced risk-factors affect the returns on options more than the risk-free rate.

\section{Conclusions}

In the recent empirical option pricing literature, several studies proposed extensions of the Black and Scholes model based on a deterministic volatility process for the underlying assets. These models are attractive for several reasons. First, in this class of models the market is dynamically complete, so that derivative securities can be priced by no-arbitrage using standard replication arguments. There is no need for general equilibrium models to characterize the behavior of the risk premia and therefore no need to estimate them. Second, in some of these models the implied state price density can be extrapolated in a way that is exactly consistent with observed option prices.

In this paper we study the dynamic implications of this class of models. Instead of running a horse race in terms of the dollar value of the pricing errors of competing models, we construct a statistical test of the main testable restriction of deterministic volatility models, namely, that options are redundant securities. Two questions are asked. First, do the returns on the underlying and the at-the-money option span the prices in the economy, or do we need additional information from other option returns or the risk-free rate? Second, is there any difference before and after the 1987 crash in terms of the spanning properties of the option market?

We cast these questions into martingale restrictions on the pricing kernel. Testing our hypotheses within this framework has several advantages. First, we can ask not only if volatility depends 
on additional risk-factors apart from the underlying, but also if these risk-factors are priced in equilibrium. Second, we can avoid any distributional assumptions on the process of the underlying since our tests are semi-parametric. Third, we do not need to make exogenous assumptions on the structure of the measurement errors in order to obtain asymptotic test statistics. Also, we take into account conditional information and we utilize both the cross-sectional and the time-series dimension of option returns.

In terms of results, we present three main findings. First, all our tests suggest that the returns of the in- and out-of-the-money options are needed for spanning purposes. These findings suggest that returns on away-from-the-money options are driven by different economic factors than those relevant for at-the-money options. These differences became stronger since the 1987 crash, while options appear to be redundant assets before the 1987 crash. Some authors have suggested that away-from-the-money options are typically used by a different clientele than their at-the-money counterparts, an example being portfolio insurers. Our empirical results would be consistent with such explanation.

Second, when using the model-independent $\delta$-metric and running test $V I I I_{c}$ conditionally (model 4) the risk-free rate is redundant in all subsamples. This suggests that accounting for stochastic interest rates might not help too much in explaining option returns. Consequently, modeling some other risk-factor, such as stochastic volatility or jumps, might be more beneficial.

Third, a comparison between the tests based on the model-dependent GMM metric and the model-independent $\delta$-metric underlines the importance of running robustness checks with respect to the metric used. The risk-free rate is always redundant in the pre-crash period. The result is robust to different specifications of the test. However, in the post-crash period the GMM test rejects the null hypothesis of redundancy. This result is not confirmed when the $\delta$-metric is used, thus suggesting that the deterministic term structure of interest rates is not the most restrictive assumption in order to price and hedge options.

Overall, these findings indicate that an important dimension of the dynamics of option prices that is relevant for dynamic hedging strategies is not captured by deterministic volatility models. As a practical implication, models need to incorporate additional priced risk-factors in order to price exotic derivatives, formulate a efficient portfolios, and construct hedges and dynamic risk management strategies. 


\section{Appendix: The Tests}

If $\beta_{r}$ is the restricted set of parameters, we extend the results of Jagannathan and Wang (1997) to show that:

Asymptotic Properties of Test 3 ( $\delta$ metric). Let $D_{T}=\frac{1}{T} R^{\prime} Y$ and $\tilde{D}_{T}=\frac{1}{T} R^{\prime} Y_{r}$, where $Y_{r}$ is the $\left(T \times k_{r}\right)$ restricted matrix of pricing factors that are presumed to span the pricing kernel, and $D_{T} \rightarrow D, \tilde{D}_{T} \rightarrow \tilde{D}$. Then:

$$
\begin{gathered}
T\left[d_{T}^{2}\left(\hat{\beta}_{r}\right)-d_{T}^{2}(\hat{\beta})\right] \rightarrow \sum_{i=1}^{k-k_{r}} \lambda_{i} \chi_{(1)}^{2} \\
\lambda=e i g\left(\left(W^{1 / 2}\right)^{\prime} Q^{-1 / 2}\left[D\left(D^{\prime} Q^{-1} D\right)^{-1} D^{\prime}-\tilde{D}\left(\tilde{D}^{\prime} Q^{-1} \tilde{D}\right)^{-1} \tilde{D}^{\prime}\right] Q^{-1 / 2} W^{1 / 2}\right)
\end{gathered}
$$

with $W^{1 / 2}$ being the lower triangular Cholesky decomposition of the asymptotic covariance matrix of the pricing errors $\sqrt{T}\left[h_{T}\left(\beta_{0}\right)\right]$, under the null hypothesis that the model is correctly specified and with eig defining the vector of eigenvalues.

Within the vector of eigenvalues $\lambda$ only $\left(k-k_{r}\right)$ eigenvalues are nonzero. The asymptotic distribution is a weighted sum of $\left(k-k_{r}\right)$ chi-square distributions with one degree of freedom.

Proof: We define $d^{2}(\beta)$ to be the maximum least square population distance:

$$
d^{2}(\beta)=E\left[h_{t}(\beta)\right]^{\prime} Q^{-1} E\left[h_{t}(\beta)\right]
$$

with $Q=E\left[R_{t}^{\prime} R_{t}\right]$. The sample counterpart of this measure is:

$$
d_{T}^{2}(\beta)=\left[\iota-D_{T} \beta\right]^{\prime} Q_{T}^{-1}\left[\iota-D_{T} \beta\right]
$$

where $D_{T}=\frac{1}{T} \underset{(n \times T)(T \times k)}{R_{T}^{\prime}} \underset{T}{Y_{T}}$, with $Y_{T}$ being the matrix of pricing factors that are presumed to span the space of returns, converge asymptotically to $D$ and $Q_{T} \rightarrow Q$. Under the null hypothesis that the model is correctly specified and $\beta=\beta_{o}, \sqrt{T} h_{T}\left(\beta_{o}\right)$ is distributed $N(0, W)$. The metric $Q$ is 
therefore not "optimal" in the GMM sense as $Q^{-1} \neq W^{-1}$. From the first order conditions and the mean value theorem, it is easy to show that:

$$
\hat{\beta}_{T}=\left(D_{T}^{\prime} Q_{T}^{-1} D_{T}\right)^{-1} D_{T}^{\prime} Q_{T}^{-1} \iota
$$

and

$$
\sqrt{T} h_{T}\left(\hat{\beta}_{T}\right)=\left[I_{n}-D_{T}\left(D_{T}^{\prime} Q_{T}^{-1} D_{T}\right)^{-1} D_{T}^{\prime} Q_{T}^{-1}\right] \sqrt{T} h_{T}\left(\beta_{o}\right)
$$

Let $\eta$ be $N(0, I)$, then if we define $W^{1 / 2}$ to be the lower triangular Cholesky decomposition of $W$ so that $W=\left(W^{1 / 2}\right)\left(W^{1 / 2}\right)^{\prime}$, we have:

$$
\begin{aligned}
\sqrt{T} h_{T}\left(\hat{\beta}_{T}\right) & =\left[I_{n}-D_{T}\left(D_{T}^{\prime} Q_{T}^{-1} D_{T}\right)^{-1} D_{T}^{\prime} Q_{T}^{-1}\right] W^{1 / 2} \eta \\
& =P W^{1 / 2} \eta
\end{aligned}
$$

Since $d_{T}^{2}\left(\hat{\beta}_{T}\right)=h_{T}\left(\hat{\beta}_{T}\right)^{\prime} Q_{T}^{-1} h_{T}\left(\hat{\beta}_{T}\right)$, then:

$$
d_{T}^{2}\left(\hat{\beta}_{T}\right)=\eta^{\prime}\left(W^{1 / 2}\right)^{\prime}\left[Q_{T}^{-1}-Q_{T}^{-1} D_{T}\left(D_{T}^{\prime} Q_{T}^{-1} D_{T}\right)^{-1} D_{T}^{\prime} Q_{T}^{-1}\right] W^{1 / 2} \eta
$$

Define $Q^{-1 / 2}$ to be the lower triangular Cholesky decomposition of $Q^{-1}$ so that $Q^{-1}=\left(Q^{-1 / 2}\right)\left(Q^{-1 / 2}\right)^{\prime}$, we have:

$$
\begin{aligned}
& d_{T}^{2}\left(\hat{\beta}_{T}\right)=\left[\eta^{\prime}\left(W^{1 / 2}\right)^{\prime} Q_{T}^{-1 / 2}\right] M\left[\left(Q_{T}^{-1 / 2}\right)^{\prime} W^{1 / 2} \eta\right] \\
& M=\left[I_{n}-\left(Q_{T}^{-1 / 2}\right)^{\prime} D_{T}\left(D_{T}^{\prime} Q_{T}^{-1} D_{T}\right)^{-1} D_{T}^{\prime} Q_{T}^{-1 / 2}\right]
\end{aligned}
$$

Observe that $M$ is idempotent and it has $k$ linearly dependent components since:

$$
\underset{k \times n}{D_{T}^{\prime}} Q_{T}^{-1 / 2} M=0
$$

so that $\left[\left(W^{1 / 2}\right)^{\prime} Q_{T}^{-1 / 2}\right] M\left[\left(Q_{T}^{-1 / 2}\right)^{\prime} W^{1 / 2}\right]$ has $(n-k)$ non-zero eigenvalues $\lambda_{i}$. Let $H$ be the orthogonal matrix of eigenvector associated to the diagonal eigenvalue matrix $\Lambda$, so that $\left[\left(W^{1 / 2}\right)^{\prime} Q_{T}^{-1 / 2}\right]$ $M\left[\left(Q_{T}^{-1 / 2}\right)^{\prime} W^{1 / 2}\right]=H^{\prime} \Lambda H$. Let $\tilde{\eta}=H^{\prime} \eta$, from the orthogonality of $H$, it follows that $\tilde{\eta}$ is distributed $N(0, I)$, so that:

$$
T d_{T}^{2}\left(\hat{\beta}_{T}\right) \rightarrow \sum_{i=1}^{n-k} \lambda_{i} \chi_{(1)}^{2}
$$

Consider now the restricted model:

$$
\tilde{d}_{T}^{2}(\beta)=\left[\iota-\tilde{D}_{T} \beta\right]^{\prime} Q_{T}^{-1}\left[\iota-\tilde{D}_{T} \beta\right]
$$


where $\tilde{D}_{T}=\frac{1}{T} R_{T}^{\prime} \tilde{Y}_{T}$, with $\tilde{Y}_{T}$ being the restricted matrix of $k_{r}$ pricing factors that are presumed to span the space of returns. Under the null hypothesis that the restrictions are satisfied:

$$
\begin{aligned}
\sqrt{T} h_{T}\left(\tilde{\beta}_{T}\right) & =\tilde{P} W^{1 / 2} \eta \\
\tilde{P} & =\left[I_{n}-\tilde{D}_{T}\left(\tilde{D}_{T}^{\prime} Q_{T}^{-1} \tilde{D}_{T}\right)^{-1} \tilde{D}_{T}^{\prime} Q_{T}^{-1}\right]
\end{aligned}
$$

$P$ has $n-k_{r}$ non-zero eigenvalues, as $\tilde{D}_{T}^{\prime} Q_{T}^{-1} P=0$.

Consider now the difference between the two LS distances, under the null that $n-k_{r}$ pricing factors do not span the pricing kernel:

$$
\begin{aligned}
& \sqrt{T}\left[h_{T}\left(\tilde{\beta}_{T}\right)-h_{T}\left(\hat{\beta}_{T}\right)\right]=(\tilde{P}-P) \sqrt{T} h_{T}\left(\beta_{o}\right) \\
&=(\tilde{P}-P) W_{L}^{1 / 2} \eta \\
& T\left[d_{T}^{2}\left(\tilde{\beta}_{T}\right)-d_{T}^{2}\left(\hat{\beta}_{T}\right)\right]=T\left[h_{T}\left(\tilde{\beta}_{T}\right)-h_{T}\left(\hat{\beta}_{T}\right)\right]^{\prime} Q^{-1}\left[h_{T}\left(\tilde{\beta}_{T}\right)-h_{T}\left(\hat{\beta}_{T}\right)\right]
\end{aligned}
$$

Observe that:

$$
\left(Q^{-1 / 2}\right)^{\prime}[\tilde{P}-P]=[\tilde{M}-M]\left(Q^{-1 / 2}\right)^{\prime}
$$

so that:

$$
T\left[d_{T}^{2}\left(\tilde{\beta}_{T}\right)-d_{T}^{2}\left(\hat{\beta}_{T}\right)\right]=\eta^{\prime}\left[\left(W^{1 / 2}\right)^{\prime} Q^{-1 / 2}\right](\tilde{M}-M)\left[\left(Q^{-1 / 2}\right)^{\prime} W^{1 / 2}\right] \eta
$$

Observe that if $\tilde{M}$ and $M$ are idempotents, then also $\tilde{M}-M$ is idempotent with $\left(n-k_{r}\right)-(n-k)$ nonzero elements, so that $\left[\left(W^{1 / 2}\right)^{\prime} Q^{-1 / 2}\right](\tilde{M}-M)\left[\left(Q^{-1 / 2}\right)^{\prime} W^{1 / 2}\right]$ has $\left(k-k_{r}\right)$ non-zero eigenvalues $\lambda_{i}^{*}$. Let $H^{*}$ be the orthogonal matrix of eigenvectors associated to the diagonal eigenvalue matrix $\Lambda^{*}$, so that:

$$
\left[\left(W^{1 / 2}\right)^{\prime} Q^{-1 / 2}\right](\tilde{M}-M)\left[\left(Q^{-1 / 2}\right)^{\prime} W^{1 / 2}\right]=H^{*^{\prime}} \Lambda^{*} H^{*}
$$

Let $\eta^{*}=H^{* \prime} \eta$, from the orthogonality of $H^{*}$, it follows that $\eta^{*}$ is distributed $N(0, I)$, so that:

$$
T\left[d_{T}^{2}\left(\tilde{\beta}_{T}\right)-d_{T}^{2}\left(\hat{\beta}_{T}\right)\right] \rightarrow \sum_{i=1}^{k-k_{o}} \lambda_{i}^{*} \chi_{(1)}^{2}
$$




\section{References}

[1] Ait-Sahalia, Y., and A. W. Lo, 1998, "Nonparametric Estimation of State-Price Densities Implicit in Financial Asset Prices," Journal of Finance, 53, 499-547.

[2] Amin, K. I., and R. A. Jarrow, 1991, "Pricing Foreign Currency Options under Stochastic Interest Rates," Journal of International Money and Finance, 10, 310-329.

[3] Andrews, D. W. K., 1991, "Heteroskedasticity and Autocorrelation Consistent Covariance Matrix Estimation," Econometrica, 59, 817-858.

[4] Bakshi, G., C. Cao, and Z. Chen, 1997, "Empirical Performance of Alternative Option Pricing Models," Journal of Finance, 52, 2003-2049.

[5] Bates, D. S., 1991, "The Crash of '87: Was it Expected? The Evidence From Options Markets," Journal of Finance, 46, 1009-1044.

[6] Bates, D. S., 1998, "Post-'87 Crash Fears in the S\&P500 Futures Option Market," working paper, University of Iowa. Journal of Econometrics, forthcoming.

[7] Bekaert, G., and M. S. Urias, 1996, "Diversification, Integration and Emerging Markets ClosedEnd Funds," Journal of Finance, 51, 835-869.

[8] Black, F., and M. Scholes, 1973, "The Pricing of Options and Corporate Liabilities," Journal of Political Economy, 81, 637-654.

[9] Brenner, M., and Y. H. Eom, 1997, "No-Arbitrage Option Pricing: New Evidence on the Validity of the Martingale Property," working paper, New York University.

[10] Braun, P. A., 1992, "Test of Consistent Pricing and No Arbitrage," working paper, Kellogg Graduate School of Management, Northwestern University.

[11] Chamberlain, G. and M. Rothschild, 1983, "Arbitrage, Factor Structure, and Mean-Variance Analysis on Large Asset Markets," Econometrica, 51, 1281-1304.

[12] Cochrane, J. H., and L. P. Hansen, 1992, "Asset Pricing Explorations for Macroeconomics," NBER Macroeconomics Annual 1992, MIT Press, Cambridge, 115-165. 
[13] Cox, J. C., and S. A. Ross, 1976, "The Valuation of Options for Alternative Stochastic Processes," Journal of Financial Economics, 3, 145-166.

[14] Derman, E., and I. Kani, 1994, "Riding on the Smile," Risk, 7, 32-39.

[15] DeSantis, G., 1993, "Volatility Bounds for Stochastic Discount Factors: Tests and Implications from International Stock Returns," Ph.D. dissertation, University of Chicago.

[16] Dumas, B., J. Fleming, and R. E. Whaley, 1998, "Implied Volatility Functions: Empirical Tests," Journal of Finance, 53, 2059-2106.

[17] Dupire, B., 1994, "Pricing with a Smile," Risk, 7, 18-20.

[18] Fleming, J., B. Ostdiek, and R. E. Whaley, 1995, "Predicting Stock Market Volatility: a New Measure," Journal of Futures Markets, 15, 265-302.

[19] Gemmill, G., and N. Kamiyama, 1997, "International Transmissions of Option Volatility and Skewness: When you're Smiling, does the Whole World Smile?", working paper, City University of London.

[20] Gruenbichler, A., and F. A. Longstaff, 1996, "Valuing Futures and Options on Volatility," Journal of Banking and Finance, 20, 985-1001.

[21] Hansen, L. P., 1982, "Large Sample Properties of Generalized Method of Moments Estimators," Econometrica, 50, 1029-1054.

[22] Hansen, L. P., and Ravi Jagannathan, 1997, "Assessing Specification Errors in Stochastic Discount Factor Models," Journal of Finance, 52, 557-590.

[23] Hansen, L. P., and S. F. Richard, 1987, "The Role of Conditioning Information in Deducing Testable Restrictions Implied by Dynamic Asset Pricing Models," Econometrica, 55, 587-614.

[24] Harrison, J. M., and D. M. Kreps, 1979, "Martingales and Arbitrage in Multiperiod Securities Markets," Journal of Economic Theory, 20, 381-408.

[25] Heston, S. L., 1993, "A Closed Form Solution for Options with Stochastic Volatility with Applications to Bonds and Currency Options," Review of Financial Studies, 6, 327-343. 
[26] Hull, J. C. and A. White, 1987, "The Pricing of Options with Stochastic Volatilities," Journal of Finance, 42, 281-300.

[27] Jackwerth, J. C., and M. Rubinstein, 1998, "Recovering Stochastic Processes from Options Prices," working paper, University of California at Berkeley.

[28] Jagannathan, R., and Z. Wang, 1997, "The Conditional CAPM and the Cross-Section of Expected Returns," working paper, Federal Reserve Bank of Minneapolis, Research Department Staff Report 208.

[29] Knez, P. J., 1993, "Pricing Money Market Securities with Stochastic Discount Factors," working paper, University of Wisconsin.

[30] Longstaff, F. A., 1995, "Option Pricing and the Martingale Restriction," Review of Financial Studies, 8, 1091-1124.

[31] Madan, D., and E. Chang, 1996, "The VG Option Pricing Model," working paper, University of Maryland and Georgia Institute of Technology.

[32] Melino, A., and S. M. Turnbull, 1990, "Pricing Foreign Currency Options with Stochastic Volatility," Journal of Econometrics, 45, 239-265.

[33] Melino, A., and S. M. Turnbull, 1995, "Misspecification and the Pricing and Hedging of LongTerm Foreign Currency Options," Journal of International Money and Finance, 14, 373-393.

[34] Merton, R. C., 1973, "Theory of Rational Option Pricing," Bell Journal of Economics, 4, $141-183$.

[35] Merton, R. C., 1976, "Option Pricing when Underlying Stock Returns are Discontinuous," Journal of Financial Economics, 3, 125-144.

[36] Newey, W. K., and K. D. West, 1987, "A Simple, Positive-Semidefinite, Heteroskedasticity and Autocorrelation Consistent Covariance Matrix," Econometrica, 55, 703-708.

[37] Roll, R., 1984, "A Simple Implicit Measure of the Effective Bid-Ask Spread in an Efficient Market," Journal of Finance, 39, 1127-39. 
[38] Rubinstein, M., 1985, "Nonparametric Tests of Alternative Option Pricing Models Using All Reported Trades and Quotes on the 30 Most Active CBOE Option Classes from August 23, 1976 through August 31, 1978," Journal of Finance, 40, 455-480.

[39] Rubinstein, M., 1994, "Implied Binomial Trees," Journal of Finance, 49, 771-818.

[40] Scott, L. O., 1987, "Option Pricing when the Variance Changes Randomly: Theory, Estimation, and an Application," Journal of Financial and Quantitative Analysis, 22, 419-438.

[41] Stein, E. M., and J. C. Stein, 1991, "Stock Price Distributions with Stochastic Volatility: an Analytic Approach," Review of Financial Studies, 4, 727-752.

[42] Wiggins, J. B., 1987, "Option Values under Stochastic Volatility: Theory and Empirical Estimates," Journal of Financial Economics, 19, 351-372. 
Table 1

Trading Volumes ${ }^{a}$

\begin{tabular}{|c|c|c|c|c|c|c|c|}
\hline \multirow[t]{2}{*}{ year } & \multicolumn{7}{|c|}{ moneyness intervals } \\
\hline & $<0.95$ & $0.95-0.97$ & $0.97-0.99$ & $0.99-1.01$ & $1.01-1.03$ & $1.03-1.05$ & $>1.05$ \\
\hline 1986 & 18 & 13 & 18 & 22 & 15 & 13 & 16 \\
\hline 1987 & 55 & 26 & 39 & 45 & 28 & 19 & 34 \\
\hline 1988 & 15 & 6 & 11 & 20 & 16 & 12 & 24 \\
\hline 1989 & 46 & 18 & 32 & 50 & 35 & 20 & 20 \\
\hline 1990 & 81 & 32 & 47 & 82 & 60 & 39 & 80 \\
\hline 1991 & 94 & 47 & 79 & 105 & 57 & 34 & 38 \\
\hline 1992 & 77 & 50 & 77 & 99 & 66 & 39 & 31 \\
\hline 1993 & 101 & 78 & 135 & 182 & 125 & 92 & 30 \\
\hline 1994 & 133 & 113 & 191 & 317 & 215 & 130 & 86 \\
\hline 1995 & 194 & 119 & 180 & 263 & 146 & 63 & 32 \\
\hline
\end{tabular}


Table $\mathbf{2}_{a}$

\section{Sample Statistics ${ }^{b}$}

\begin{tabular}{lccccc}
\hline & & & & & \\
$R_{s}$ & mean & stddev & $\rho_{1}$ & $\rho_{2}$ & $\rho_{3}$ \\
$R_{f}$ & 2 & 86 & 0.0865 & -0.0234 & -0.0509 \\
$R_{0.96}$ & 25 & 1217 & 0.0658 & -0.0229 & -0.0012 \\
$R_{0.98}$ & 5 & 1467 & 0.0696 & -0.0152 & -0.0020 \\
$R_{1.00}$ & 3 & 1832 & 0.0825 & 0.0008 & 0.0017 \\
$R_{1.02}$ & 79 & 2412 & 0.0696 & 0.0187 & 0.0035 \\
$R_{1.04}$ & 351 & 3431 & 0.0411 & 0.0437 & 0.0173 \\
& & & & & \\
\hline \hline
\end{tabular}


Table $\mathbf{2}_{b}$

\section{Coefficients of Correlation ${ }^{c}$}

\begin{tabular}{|c|c|c|c|c|c|c|c|}
\hline & $R_{s}$ & $R_{f}$ & $R_{0.96}$ & $R_{0.98}$ & $R_{1.00}$ & $R_{1.02}$ & $R_{1.04}$ \\
\hline$R_{s}$ & 1 & 0.0031 & 0.9415 & 0.9288 & 0.9039 & 0.8519 & 0.7364 \\
\hline$R_{f}$ & & 1 & -0.0358 & -0.0361 & -0.0302 & -0.0383 & -0.0515 \\
\hline$R_{0.96}$ & & & 1 & 0.9945 & 0.9791 & 0.9410 & 0.8284 \\
\hline$R_{0.98}$ & & & & 1 & 0.9916 & 0.9593 & 0.8478 \\
\hline$R_{1.00}$ & & & & & 1 & 0.9798 & 0.8766 \\
\hline$R_{1.02}$ & & & & & & 1 & 0.9148 \\
\hline$R_{1.04}$ & & & & & & & 1 \\
\hline
\end{tabular}


Table 3

Unconditional Martingale Tests (GMM) ${ }^{d}$

\begin{tabular}{|c|c|c|c|}
\hline & \multicolumn{3}{|c|}{ Test $I I I_{a}: R_{a}^{*}=\left[R_{s}, R_{f}\right]$} \\
\hline & Pre-1987 Crash & Post-1987 Crash & Overall Sample \\
\hline$d_{T}$-value & 2.15 & 173.07 & 83.83 \\
\hline \multirow[t]{3}{*}{ p-value } & $34 \%$ & $<1 \%$ & $<1 \%$ \\
\hline & \multicolumn{3}{|c|}{ Test $I I I_{b}: R_{a}^{*}=\left[R_{s}, R_{1.00}\right] \quad R_{b}^{*}=\left[R_{0.96}, R_{1.04}\right]$} \\
\hline & Pre-1987 Crash & Post-1987 Crash & Overall Sample \\
\hline$d_{T}$-value & 39.44 & 172.00 & 148.17 \\
\hline \multirow[t]{3}{*}{ p-value } & $<1 \%$ & $<1 \%$ & $<1 \%$ \\
\hline & \multicolumn{3}{|c|}{ Test $I I I_{c}: R_{a}^{*}=\left[R_{s}, R_{1.00}\right] \quad R_{b}^{*}=\left[R_{f}\right]$} \\
\hline & Pre-1987 Crash & Post-1987 Crash & Overall Sample \\
\hline$d_{T}$-value & 0.15 & 63.46 & 34.84 \\
\hline p-value & $70 \%$ & $<1 \%$ & $<1 \%$ \\
\hline
\end{tabular}


Table 4

Conditional Martingale Tests (GMM) ${ }^{e}$

\begin{tabular}{|c|c|c|c|}
\hline \multirow[b]{2}{*}{ MOdEL 1} & \multicolumn{2}{|c|}{ Test $I V_{a}: R_{a}^{*}=\left[R_{s}, R_{f}\right]$} & $R_{b}^{*}=\left[R_{0.96}, R_{1.04}\right]$ \\
\hline & Pre-1987 Crash & Post-1987 Crash & Overall Sample \\
\hline$d_{T}-$ value & 2.60 & 236.50 & 152.21 \\
\hline p-value & $63 \%$ & $<1 \%$ & $<1 \%$ \\
\hline \multicolumn{4}{|l|}{ MODEL 2} \\
\hline$d_{T}-$ value & 1.45 & 240.57 & 210.24 \\
\hline p-value & $96 \%$ & $<1 \%$ & $<1 \%$ \\
\hline \multicolumn{4}{|l|}{ MODEL 3} \\
\hline$d_{T}-$ value & 1.27 & 191.00 & 86.06 \\
\hline p-value & $87 \%$ & $<1 \%$ & $<1 \%$ \\
\hline \multicolumn{4}{|l|}{ MODEL 4} \\
\hline$d_{T}-$ value & 2.71 & 190.58 & 77.23 \\
\hline p-value & $84 \%$ & $<1 \%$ & $<1 \%$ \\
\hline
\end{tabular}


Table 4, continue

\section{Conditional Martingale Tests (GMM)}

Test $I V_{b}: R_{a}^{*}=\left[R_{s}, R_{1.00}\right] \quad R_{b}^{*}=\left[R_{0.96}, R_{1.04}\right]$

MODEL 1

$d_{T}$-value

p-value

Model 2

$$
d_{T-v a l u e}
$$$$
\text { p-value }
$$

Model 3

$$
\begin{gathered}
d_{T-\text { value }} \\
\text { p-value }
\end{gathered}
$$

Model 4

$$
d_{T \text {-value }}
$$$$
\text { p-value }
$$

Model 1

$$
\begin{gathered}
d_{T-\text {-value }} \\
\text { p-value }
\end{gathered}
$$

Model 2

$$
\begin{gathered}
d_{T} \text {-value } \\
\text { p-value }
\end{gathered}
$$

Model 3

$$
\begin{gathered}
d_{T} \text {-value } \\
\text { p-value }
\end{gathered}
$$

Model 4

$$
\begin{gathered}
d_{T} \text {-value } \\
\text { p-value }
\end{gathered}
$$

38.85

$<1 \%$
269.03

$<1 \%$

$<1 \%$

189.09

175.13

$<1 \%$

$<1 \%$

$<1 \%$

35.60

202.24

183.57

$<1 \%$

$<1 \%$

$<1 \%$

Test $I V_{c}: R_{a}^{*}=\left[R_{s}, R_{1.00}\right] \quad R_{b}^{*}=\left[R_{f}\right]$

\begin{tabular}{ccc}
\hline Pre-1987 Crash & Post-1987 Crash & Overall Sample \\
0.66 & 110.02 & 81.04 \\
$71 \%$ & $<1 \%$ & $<1 \%$
\end{tabular}

0.06

108.67

110.41

$99 \%$

$<1 \%$

$<1 \%$

0.94

65.82

36.75

$63 \%$

$<1 \%$

$<1 \%$

0.002

63.86

44.49

$100 \%$

$<1 \%$

$<1 \%$ 


\section{Table 5}

Estimation of Bias in Test Statistics $f$

\section{Empirical Rejection Frequency}

\begin{tabular}{|c|c|c|c|c|}
\hline Test A: & $\begin{aligned} R_{a}^{*} & =\left[R_{s}, R_{1.00}\right] \\
R_{b}^{*} & =\left[R_{0.98}, R_{1.02}\right]\end{aligned}$ & Test B: & $\begin{aligned} R_{a}^{*} & =\left[R_{s}, R_{1.00}\right] \\
R_{b}^{*} & =\left[R_{f}\right]\end{aligned}$ & $p-$ value \\
\hline & $3.45 \%$ & & $4.02 \%$ & $5 \%$ \\
\hline
\end{tabular}




\section{Table 6}

\section{Bias in Test Statistics Due to Observation Error ${ }^{g}$}

\begin{tabular}{|c|c|c|c|c|}
\hline \multirow[t]{4}{*}{$\sigma_{\varepsilon}$} & \multirow[t]{4}{*}{$\rho$} & \multicolumn{2}{|c|}{ Rejection Frequency } & \multirow[t]{4}{*}{$p$-value } \\
\hline & & Test A & Test B & \\
\hline & & $R_{a}^{*}=\left[R_{s}, R_{1.00}\right]$ & $R_{a}^{*}=\left[R_{s}, R_{1.00}\right]$ & \\
\hline & & $R_{b}^{*}=\left[R_{0.98}, R_{1.02}\right]$ & $R_{b}^{*}=\left[R_{f}\right]$ & \\
\hline $6 \%$ & 0 & $5.32 \%$ & $7.64 \%$ & $5 \%$ \\
\hline $6 \%$ & $\frac{1}{2}$ & $5.36 \%$ & $5.36 \%$ & $5 \%$ \\
\hline $6 \%$ & 1 & $6.63 \%$ & $4.76 \%$ & $5 \%$ \\
\hline Average Bias & & $0.77 \%$ & $0.92 \%$ & \\
\hline
\end{tabular}


Table 7

Unconditional Martingale Tests $(\delta \text { metric })^{h}$

\begin{tabular}{|c|c|c|c|}
\hline \multirow[b]{3}{*}{$\tilde{\delta}_{T^{-v a l u e}}^{2}$} & \multicolumn{3}{|c|}{ Test $V I I_{a}: R_{a}^{*}=\left[R_{s}, R_{f}\right] \quad R_{b}^{*}=\left[R_{0.96}, R_{1.04}\right]$} \\
\hline & Pre-1987 Crash & Post-1987 Crash & Overall Sample \\
\hline & 0.34 & 189.00 & 84.41 \\
\hline \multirow[t]{3}{*}{ p-value } & $87 \%$ & $<1 \%$ & $<1 \%$ \\
\hline & \multicolumn{3}{|c|}{ Test $V I I_{b}: R_{a}^{*}=\left[R_{s}, R_{1.00}\right] \quad R_{b}^{*}=\left[R_{0.96}, R_{1.04}\right]$} \\
\hline & Pre-1987 Crash & Post-1987 Crash & Overall Sample \\
\hline$\tilde{\delta}_{T}^{2}$-value & 25.16 & 217.73 & 244.58 \\
\hline \multirow[t]{3}{*}{ p-value } & $<1 \%$ & $<1 \%$ & $<1 \%$ \\
\hline & \multicolumn{3}{|c|}{ Test $V I I_{c}: R_{a}^{*}=\left[R_{s}, R_{1.00}\right] \quad R_{b}^{*}=\left[R_{f}\right]$} \\
\hline & Pre-1987 Crash & Post-1987 Crash & Overall Sample \\
\hline$\tilde{\delta}_{T}^{2}$-value & 0.17 & 20.48 & 6.14 \\
\hline p-value & $51 \%$ & $<1 \%$ & $<1 \%$ \\
\hline
\end{tabular}


Table 8

Conditional Martingale Tests ( $\delta$ metric $)^{i}$

\begin{tabular}{|c|c|c|c|}
\hline \multirow[b]{2}{*}{ MODEL 1} & \multicolumn{2}{|c|}{ Test $V I I I_{a}: R_{a}^{*}=\left[R_{s}, R_{f}\right]$} & $R_{b}^{*}=\left[R_{0.96}, R_{1.04}\right]$ \\
\hline & Pre-1987 Crash & Post-1987 Crash & Overall Sample \\
\hline$\tilde{\delta}_{T}^{2}$-value & 0.47 & 209.93 & 159.89 \\
\hline p-value & $98 \%$ & $<1 \%$ & $<1 \%$ \\
\hline \multicolumn{4}{|l|}{ MODEL 2} \\
\hline$\tilde{\delta}_{T}^{2}$-value & 4.22 & 209.23 & 177 \\
\hline p-value & $72 \%$ & $<1 \%$ & $<1 \%$ \\
\hline \multicolumn{4}{|l|}{ MODEL 3} \\
\hline$\tilde{\delta}_{T^{-v a l u e}}^{2}$ & 3.53 & 190.79 & 84.50 \\
\hline p-value & $60 \%$ & $<1 \%$ & $<1 \%$ \\
\hline \multicolumn{4}{|l|}{ MODEL 4} \\
\hline$\tilde{\delta}_{T}^{2}$-value & 2.43 & 177.00 & 77.47 \\
\hline p-value & $91 \%$ & $<1 \%$ & $<1 \%$ \\
\hline
\end{tabular}


Table 8, continue

Conditional Martingale Tests ( $\delta$ metric)

Test $V I I I_{b}: R_{a}^{*}=\left[R_{s}, R_{1.00}\right] \quad R_{b}^{*}=\left[R_{0.96}, R_{1.04}\right]$

Model 1

$$
\begin{gathered}
\tilde{\delta}_{T}^{2} \text {-value } \\
\text { p-value }
\end{gathered}
$$

Model 2

$$
\begin{gathered}
\tilde{\delta}_{T}^{2} \text {-value } \\
\text { p-value }
\end{gathered}
$$

Model 3

$$
\begin{gathered}
\tilde{\delta}_{T}^{2} \text {-value } \\
\text { p-value }
\end{gathered}
$$

Model 4

$$
\begin{gathered}
\tilde{\delta}_{T}^{2} \text {-value } \\
\text { p-value }
\end{gathered}
$$

Model 1

$$
\tilde{\delta}_{T}^{2} \text {-value }
$$$$
\text { p-value }
$$

Model 2

$$
\begin{gathered}
\tilde{\delta}_{T}^{2} \text {-value } \\
\text { p-value }
\end{gathered}
$$

Model 3

$$
\begin{gathered}
\tilde{\delta}_{T}^{2} \text {-value } \\
\text { p-value }
\end{gathered}
$$

Model 4

$$
\begin{gathered}
\tilde{\delta}_{T}^{2} \text {-value } \\
p \text {-value }
\end{gathered}
$$

Pre-1987 Crash

27.12

$<1 \%$

27.56

$<1 \%$

27.60

230.35

265.50

$<1 \%$

$<1 \%$

$<1 \%$

24.66

220.55

264.71

$<1 \%$

$<1 \%$

$<1 \%$

Test $V I I I_{c}: R_{a}^{*}=\left[R_{s}, R_{1.00}\right] \quad R_{b}^{*}=\left[R_{f}\right]$

Pre-1987 Crash Post-1987 Crash Overall Sample

0.72

42.49

33.37

$80 \%$

$<1 \%$

$2 \%$

0.42

48.93

42.01

$98 \%$

$<1 \%$

$2 \%$

0.53

24.75

7.08

$84 \%$

$<1 \%$

$23 \%$

1.03

8.40

13.17

$91 \%$

$42 \%$

$27 \%$ 


\section{Table Notes}

a. [Table 1] Average daily trading volume in million US dollars of all put and call options with maturities spanning 45 days. Volume is expressed in terms of the notional value of the contracts.

b. [Table $2_{a}$ ] This table reports mean, standard deviation, and the first three sample autocorrelation coefficients $\rho$ of returns on the underlying asset $R_{s}$, the risk-free rate $R_{f}$, and the five option returns $R_{i}$ where $i$ indicates the moneyness level $\frac{K}{S}$. The units of measure are basis points per day.

c. $\left[\right.$ Table $\left.2_{b}\right]$ This table reports the contemporaneous correlations of returns on the underlying asset $R_{s}$, the risk-free rate $R_{f}$, and the five option returns $R_{i}$ where $i$ indicates the moneyness level $\frac{K}{S}$

d. [Table 3] We test the null hypothesis that $H_{o}: \beta_{b}=0$ in the martingale retriction:

$$
\begin{gathered}
0=E\left[1-m_{t, t+\Delta t} R_{t, t+\Delta t}\right] \\
m_{t, t+\Delta t}=\exp \left(\sum_{j} \beta_{a}^{\prime} R_{a, t_{j}}^{*}+\beta_{b}^{\prime} R_{b, t_{j}}^{*}\right)
\end{gathered}
$$

The test is based on the pricing errors $h_{t+\Delta t}=1-m_{t+\Delta t} R_{t+\Delta t}$ by forming:

$$
J_{T}=\left[\frac{1}{\sqrt{T}} \sum_{t=1}^{T} h_{t}\left(m\left(\beta, R^{*}\right), R\right)\right]^{\prime} W_{T}^{-1}\left[\frac{1}{\sqrt{T}} \sum_{t=1}^{T} h_{t}\left(m\left(\beta, R^{*}\right), R\right)\right]
$$

and computing the difference $d_{T} \equiv J_{T}\left(\beta_{a}, \beta_{b}=0 ; R\right)-J_{T}(\beta ; R)$ which is distributed under the null as a $\chi_{\left(n_{b}\right)}^{2}$, where $n_{b}$ is the number of restricted parameters. $R$ is the vector of returns on seven assets, $R=\left[R_{s}, R_{f}, R_{0.96}, R_{0.98}, R_{1.00}, R_{1.02}, R_{1.04}\right]$. $R_{s}$ is the return on the underlying asset and $R_{f}$ is the risk-free rate. The remaining five returns are on options with with levels of moneyness of $0.96,0.98,1.00,1.02$, and 1.04. $R_{t_{j}}^{*}$ are the $\log$ returns sampled at frequency $\frac{\Delta t}{n}$ in the interval $[t, t+\Delta t]$. At the first stage, we initialize the weighting matrix to be the covariance matrix of the pricing errors associated with the risk neutral pricing kernel $m_{t}=1 / r_{t}^{f}$. The final estimates are obtained at the second stage after updating the weighting matrix using a 5 lag Newey-West estimator of the covariance matrix of the first stage pricing errors.

e. [Table 4] We test the null hypothesis that $H_{o}: \beta_{b}=0$ in the following martingale retriction for the hedging errors of time varying managed portfolio of options: 


$$
\begin{gathered}
0=E\left[h_{t, t+\Delta t} \mid \mathcal{I}_{t}\right] \\
h_{t+\Delta t}=\left[\begin{array}{cc}
1 & \\
1 & \phi\left(\omega_{t}\right)
\end{array}\right]-m_{t, t+\Delta t}\left[\begin{array}{ll}
R_{t+\Delta t} & \\
R_{t+\Delta t} & \phi\left(\omega_{t}\right)
\end{array}\right] \\
m_{t, t+\Delta t}=\exp \left(\sum_{j} \beta_{t_{j}, a}^{\prime} R_{a, t_{j}}^{*}+\beta_{t, b}^{\prime} R_{b, t_{j}}^{*}\right)
\end{gathered}
$$

The parameters $\beta \in I_{t}$ are time varying and parameterized as functions of the variables $\phi\left(\omega_{t}\right)$ in the information set as $\beta_{t}=\beta^{\prime} \phi\left(\omega_{t}\right)$. The test statistics is based on:

$$
J_{T}=\left[\frac{1}{\sqrt{T}} \sum_{t=1}^{T} h_{t+\Delta t}\right]^{\prime} W_{T}^{-1}\left[\frac{1}{\sqrt{T}} \sum_{t=1}^{T} h_{t+\Delta t}\right]
$$

We compute the difference $d_{T} \equiv J_{T}\left(\beta_{a}, \beta_{b}=0 ; R\right)-J_{T}(\beta ; R)$ which is distributed, under the null, as a $\chi_{\left(n_{\omega} n_{b}\right)}^{2}$, where $n_{\omega}$ is the number of conditioning variables (including the unit vecto) and $n_{b}$ and $n_{a}$ are the number of restricted and unrestricted parameters respectively. $R$ is the vector of returns on seven assets, $R=\left[R_{s}, R_{f}, R_{0.96}, R_{0.98}, R_{1.00}, R_{1.02}, R_{1.04}\right] . R_{s}$ is the return on the underlying asset and $R_{f}$ is the risk-free rate. The remaining five returns are on options with with levels of moneyness of $0.96,0.98,1.00,1.02$, and 1.04. $R_{t_{j}}^{*}$ are the logarithimc returns sampled at frequency $\frac{\Delta t}{n}$ in the interval $[t, t+\Delta t]$. The conditioning variables are polynomials in the underlying asset, as in equation (17). At the first stage, we initialize the weighting matrix to be the covariance matrix of the pricing errors associated with the risk neutral pricing kernel $m_{t}=1 / r_{t}^{f}$. The final estimates are obtained at a second stage after updating the weighting matrix using a 5 lag Newey-West estimator of the covariance matrix of the first stage pricing errors.

f. [Table 5] We simulate 1000 days of the following CEV model:

$$
\frac{d S_{t}}{S_{t}}=(\mu-d) d t+\sigma S_{t}^{\rho-1} d W_{t}
$$

We initialize the parameters at their historical sample averages with $\mu=13.58 \%, r=5.43 \%$, $d=3.12 \%, \sigma S_{o}^{\rho-1}=16.16 \%$ with $\rho=1 / 2$. The simulated option prices have maturity of 45 days and moneyness levels equal to 0.98 and 1.02. The values reported in the table are the "Rejection Frequency" of two types of Chi-square tests. These are the the empirical frequencies at which the $\Delta J_{T}$ test statistics is higher than the Chi-square level for a $5 \%$ p-value. If the bias is zero then the "Rejection Frequency" should be $5 \%$. 
g. [Table 6] We simulate 1000 days of the following CEV model:

$$
\frac{d S_{t}}{S_{t}}=(\mu-d) d t+\sigma S_{t}^{\rho-1} d W_{t}
$$

We initialize the parameters at their historical sample averages with $\mu=13.58 \%, r=5.43 \%$, $d=3.12 \%, \sigma S_{o}^{\rho-1}=16.16 \%$. For different values of $\rho$ we simulate option prices with a maturity of 45 days and moneyness levels equal to $0.98,1.00$ and 1.02 . Let $R_{t}$ be the return on an option, and let $\hat{R}_{t}$ be the return of the option observed with measurement error

$$
\hat{R}_{t}=R_{t}+\varepsilon_{t}, \quad \varepsilon_{t} \sim i i d\left(0, \sigma_{\varepsilon}\right)
$$

The "Rejection Frequency" is the empirical frequency at which the $\Delta J_{T}$ test statistics is higher than the Chi-square level for a $5 \%$ p-value. If the bias is zero then the "Rejection frequency" should be $5 \%$.

h. [Table 7] We test the null hypothesis that $H_{o}: \beta_{b}=0$ in the martingale retriction:

$$
\begin{gathered}
0=E\left[1-m_{t, t+\Delta t} R_{t, t+\Delta t}\right] \\
m_{t, t+\Delta t}=\exp \left(\sum_{j} \beta_{a}^{\prime} R_{a, t_{j}}^{*}+\beta_{b}^{\prime} R_{b, t_{j}}^{*}\right)
\end{gathered}
$$

The test is based on the pricing errors $h_{t+\Delta t}=1-m_{t+\Delta t} R_{t+\Delta t}$ by forming:

$$
\delta_{T}^{2}=\left[\frac{1}{\sqrt{T}} \sum_{t=1}^{T} h_{t}\left(m^{*}\left(\beta, R^{*}\right), R\right)\right]^{\prime} Q_{T}^{-1}\left[\frac{1}{\sqrt{T}} \sum_{t=1}^{T} h_{t}\left(m^{*}\left(\beta, R^{*}\right), R\right)\right]
$$

with $R_{a}$ being the matrix of pricing factors that are supposed to span the pricing kernel and $Q_{T}$ being a consistent estimator of the second moment matrix of the returns $Q_{T} \rightarrow Q=E\left(R_{t}^{\prime} R_{t}\right)$. The statistics $\tilde{\delta}_{T}^{2} \equiv \delta_{T}^{2}\left(\beta_{a}, \beta_{b}=0 ; R\right)-\delta_{T}^{2}(\beta ; R)$ is shown to be distributed, under the null, as a weighted sum of $\chi_{(1)}^{2}$ and can be tabulated numerically. $R$ is the vector of returns on seven assets, $R=\left[R_{s}\right.$, $\left.R_{f}, R_{0.96}, R_{0.98}, R_{1.00}, R_{1.02}, R_{1.04}\right] . R_{s}$ is the return on the underlying asset and $R_{f}$ is the risk-free rate. The remaining five returns are on options with with levels of moneyness of 0.96, 0.98, 1.00, 1.02, and 1.04. $R_{t_{j}}^{*}$ are the logarithmic returns sampled at frequency $\frac{\Delta t}{n}$ in the interval $[t, t+\Delta t]$.

i. [Table 8] We test the null hypothesis that $H_{o}: \beta_{b}=0$ in the following martingale retriction for the hedging errors of time varying managed portfolio of options: 


$$
\begin{gathered}
0=E\left[h_{t, t+\Delta t} \mid \mathcal{I}_{t}\right] \\
h_{t+\Delta t}=\left[\begin{array}{cc}
1 & \\
1 & \phi\left(\omega_{t}\right)
\end{array}\right]-m_{t, t+\Delta t}\left[\begin{array}{cc}
R_{t+\Delta t} & \\
R_{t+\Delta t} & \phi\left(\omega_{t}\right)
\end{array}\right] \\
m_{t, t+\Delta t}=\exp \left(\sum_{j} \beta_{t_{j}, a}^{\prime} R_{a, t_{j}}^{*}+\beta_{t_{j}, b}^{\prime} R_{b, t_{j}}^{*}\right)
\end{gathered}
$$

The parameters $\beta \in I_{t}$ are time varying and parameterized as functions of the variables $\phi\left(\omega_{t}\right)$ in the information set as $\beta_{t}=\beta^{\prime} \phi\left(\omega_{t}\right)$. The test statistics is based on:

$$
\delta_{T}^{2}(\beta)=\left[\frac{1}{\sqrt{T}} \sum_{t=1}^{T} h_{t+\Delta t}\right]^{\prime} Q_{T}^{-1}\left[\frac{1}{\sqrt{T}} \sum_{t=1}^{T} h_{t+\Delta t}\right]
$$

with $R_{a}$ being the matrix of returns on the subset of assets that are conjectured to span the time varying investment opportunity set and $\phi\left(\omega_{t}\right) \in I_{t}$, and $Q_{T}$ being a consistent estimator of the second moment matrix of the returns $Q_{T} \rightarrow Q=E\left(R_{t}^{\prime} R_{t}\right)$. Defining $\tilde{\delta}_{T}^{2} \equiv \delta_{T}^{2}\left(\beta_{a}, \beta_{b}=0 ; R\right)$ $\delta_{T}^{2}(\beta ; R)$, we show that $\tilde{\delta}_{T}^{2}$ is distributed, under the null, as a weighted sum of $\chi_{(1)}^{2}$ that can be tabulated numerically. $R$ is the vector of returns on seven assets, $R=\left[R_{s}, R_{f}, R_{0.96}, R_{0.98}, R_{1.00}\right.$, $\left.R_{1.02}, R_{1.04}\right]$. $R_{s}$ is the return on the underlying asset and $R_{f}$ is the risk-free rate. The remaining five returns are on options with with levels of moneyness of $0.96,0.98,1.00,1.02$, and 1.04. $R_{t_{j}}^{*}$ are the log-returns sampled at frequency $\frac{\Delta t}{n}$ in the interval $[t, t+\Delta t]$. The conditioning variables are polynomials in the level of the underlying asset as in equation (17). 


\section{Notes}

${ }^{1}$ If $E\left(b b^{\prime} \mid \mathcal{I}\right)$ is non-singular, then the trading strategy $c$ that generates a given payoff $p$ is determined uniquely.

${ }^{2}$ The stochastic discount factor $\xi$ also characterizes the Radon-Nikodym derivative that defines the change of probability measure from the objective measure $Q$ to the risk neutral measure $\tilde{Q}$ under which all asset prices (unscaled) are martingale processes. If $Z$ is the price of a zero coupon bond, then $\frac{d \tilde{Q}}{d Q}$ is given by $E\left(\frac{d \tilde{Q}}{d Q} \mid \mathcal{I}_{t+1}\right)=\frac{\xi_{t+1}}{\xi_{t}} Z_{t, t+1}^{-1}$. The measure $\tilde{Q}$ is usually called "risk neutral" since it is such that any no-arbitrage price can be represented as $\pi_{t}\left(p_{t+1}\right)=Z_{t, t+1} E_{\tilde{Q}}\left(p_{t+1} \mid \mathcal{I}_{t}\right)$. However, this representation is less tractable from an econometric perspective since security prices can only be sampled under the objective measure $Q$.

${ }^{3}$ Since our data-set includes minute by minute quotes and trading prices, for each sample period $[t, t+\Delta t]$, we consider the subsequence of log returns $R_{t, t+\Delta t, n}^{*}=\left\{R_{t, t+\Delta t / n}^{*} ; R_{t+\Delta t / n, t+2 \Delta t / n}^{*}\right.$ ; $\left.R_{t+2 \Delta t / n, t+3 \Delta t / n}^{*} \cdots R_{t+(n-1) \Delta t / n, t+\Delta t}^{*}\right\}$, where $R_{t, t+\Delta t / n}^{*}=\ln \left(\frac{p(t+\Delta t / n)}{p(t)}\right)$, with $p$ being the price of a security. As $n \rightarrow \infty$, the pricing kernel $m_{t, t+\Delta t}$ is replicated by a dynamic trading strategy on a set of primitive securities with subperiod returns $R_{t, t+\Delta t, n}^{*}$.

${ }^{4}$ In the space $L^{2}$ of square integrable payoffs, the pricing kernel that satisfies the Principle of No Arbitrage is not unique. We denote by $\mathcal{M}$ the subspace of $\tilde{m} \in L^{2}$ that satisfies the relation $1=E(\tilde{m} p)$. 